TRANSACTIONS OF THE

AMERICAN MATHEMATICAL SOCIETY

Volume 358, Number 12, December 2006, Pages 5539-5564

S 0002-9947(06)03914-6

Article electronically published on July 20, 2006

\title{
BLASCHKE- AND MINKOWSKI-ENDOMORPHISMS OF CONVEX BODIES
}

\author{
MARKUS KIDERLEN
}

\begin{abstract}
We consider maps of the family of convex bodies in Euclidean $d$ dimensional space into itself that are compatible with certain structures on this family: A Minkowski-endomorphism is a continuous, Minkowski-additive map that commutes with rotations. For $d \geq 3$, a representation theorem for such maps is given, showing that they are mixtures of certain prototypes. These prototypes are obtained by applying the generalized spherical Radon transform to support functions. We give a complete characterization of weakly monotonic Minkowski-endomorphisms. A corresponding theory is developed for Blaschke-endomorphisms, where additivity is now understood with respect to Blaschke-addition. Using a special mixed volume, an adjoining operator can be introduced. This operator allows one to identify the class of Blaschke-endomorphisms with the class of weakly monotonic, non-degenerate and translation-covariant Minkowski-endomorphisms.

The following application is also shown: If a (weakly monotonic and) nontrivial endomorphism maps a convex body to a homothet of itself, then this body must be a ball.
\end{abstract}

\section{INTRODUCTION AND MAIN RESULTS}

1.1. Minkowski-endomorphisms. Let $\mathcal{K}^{d}$ be the family of convex bodies (nonempty, compact, convex subsets of $\mathbb{R}^{d}, d \geq 2$ ) equipped with the usual Hausdorff metric. The purpose of this note is to give representation theorems for mappings from $\mathcal{K}^{d}$ into itself which are compatible with certain structures on $\mathcal{K}^{d}$.

Definition 1.1. A mapping $\Phi: \mathcal{K}^{d} \rightarrow \mathcal{K}^{d}$ is called a Minkowski-endomorphism (of $\mathcal{K}^{d}$ ) if it satisfies the following conditions:

i) $\Phi$ is continuous,

ii) $\Phi$ is Minkowski-additive,

$$
\Phi(K+M)=\Phi K+\Phi M, \quad \text { for all } K, M \in \mathcal{K}^{d},
$$

iii) $\Phi$ is $S O_{d}$-equivariant:

$$
\Phi \circ \vartheta=\vartheta \circ \Phi, \quad \text { for all } \vartheta \in S O_{d} .
$$

Here, $S O_{d}$ denotes the group of rigid rotations in $\mathbb{R}^{d}$ at the origin $o$.

Received by the editors May 6, 2004 and, in revised form, November 22, 2004.

2000 Mathematics Subject Classification. Primary 52A20, 08A35; Secondary 43A90, 46T30.

Key words and phrases. Convex body, Minkowski-addition, Blaschke-addition, Hausdorff metric, Steiner point, mixed volume, equivariant map, monotonic, spherical distribution, generalized function, spherical harmonic, multiplier.

The majority of this note is taken from the author's doctoral thesis [11], written in German at the University of Karlsruhe, Germany.

(C)2006 American Mathematical Society Reverts to public domain 28 years from publication 
The (abtract) cone of Minkowski-endomorphisms of $\mathcal{K}^{d}$ will be denoted by $\operatorname{End}_{\mathrm{M}}\left(\mathcal{K}^{d}\right)$.

Endomorphisms of the space of convex bodies were first introduced in SCHNEIDER [15] with a slightly more restrictive definition: Instead of iii), equivariance with respect to the whole group of rigid motions is claimed. Even though this is natural from a geometric point of view, we prefer the present definition for two reasons: In Section 2.3 we will show that any Minkowski-endomorphism can be easily transformed into an endomorphism in the sense of Schneider by adding a suitable vector-valued function. The second reason to omit equivariance with respect to the translation group $T_{d}$ is the fact that interesting, geometrically motivated examples can now be treated in the framework of Minkowski-endomorphisms; see Example 1.2, below.

A Minkowski-endomorphism $\Phi$ is called symmetric if $\Phi(-K)=\Phi K$ holds for all $K \in \mathcal{K}^{d}$. It is called monotonic if it satisfies

$$
K \subset M \Rightarrow \Phi K \subset \Phi M
$$

for all $K, M \in \mathcal{K}^{d}$. This condition is rather strong. Consider for example the mapping $\Phi_{0}: K \mapsto K-s(K)$, where $s(K)$ is the Steiner point of $K \in \mathcal{K}^{d}$. $\Phi_{0}$ is a Minkowski-endomorphism, but it is not monotonic. $\Phi \in \operatorname{End}_{\mathrm{M}}\left(\mathcal{K}^{d}\right)$ will be called weakly monotonic if (1.1) holds for all $K, M \in \mathcal{K}^{d}$ whose Steiner points coincide with the origin. Obviously, $\Phi_{0}$ is weakly monotonic. In Section 2.3 the reader will find more details about properties of the Steiner point map $K \mapsto s(K)$ and the support function $h(K, \cdot)$, which will be needed throughout the paper. Note that $h(K, \cdot)$ will always be considered as a function on the Euclidean unit sphere $S^{d-1}$ of $\mathbb{R}^{d}$. Thus, $h(K, \cdot)$ is an element of the Banach space $\mathcal{C}\left(S^{d-1}\right)$ of continuous functions on the sphere, supplied with the maximum norm. Throughout the following we make use of the fact that every convex body is uniquely determined by its support function.

Example 1.2. Let $\mathcal{L}_{k}^{d}, k \in\{1, \ldots, d-1\}$, be the compact manifold of all $k$ dimensional linear subspaces of $\mathbb{R}^{d}$. The unique rotation invariant probability measure on this manifold will be denoted by $\nu_{k}$. The $k$-th projection mean $P_{k}(K) \in \mathcal{K}^{d}$ of a convex body $K$ is defined by

$$
h\left(P_{k}(K), \cdot\right)=\int_{\mathcal{L}_{k}^{d}} h(K \mid L, \cdot) \nu_{k}(d L),
$$

where the orthogonal projection $K \mid L$ of $K$ on $L$ is a (lower-dimensional) element of $\mathcal{K}^{d}$. The mapping $K \mapsto P_{k}(K)$ is a monotonic Minkowski-endomorphism.

The mapping $P_{d-1}$ was first considered by SchneIder [17. Injectivity issues for $P_{k}$ are addressed in Spriestersbach 21], Goodey [6], Goodey and Jiang 8], and KIDERLEN [12.

Further examples of Minkowski-endomorphisms can be found in [15]. In Section 2.3. we will recall a result from SchNEIDER [16 stating that for $d=2$, all Minkowski-endomorphisms can be obtained by "mixing" the simple endomorphisms $K \mapsto \vartheta K, \vartheta \in S O_{2}$, with respect to a measure on $S_{2}$. The main theorem of this paper shows that this result has an analogue in higher dimensions: Any $\Phi \in \operatorname{End}_{\mathrm{M}}\left(\mathcal{K}^{d}\right), d \geq 3$, is a mixture of certain prototypical endomorphisms. These prototypes can be conveniently defined using the generalized spherical Radon transform $R_{\alpha}, \alpha \in[-1,1]$, which maps $\mathcal{C}\left(S^{d-1}\right)$ into itself. For $f \in \mathcal{C}\left(S^{d-1}\right)$ and $u \in S^{d-1},\left(R_{\alpha} f\right)(u)$ is the average of the $f$-values on the subsphere $S^{d-1} \cap\left(\alpha u+u^{\perp}\right)$. 
We will show (Proposition 3.2) that for all $K \in \mathcal{K}^{d}$ and $\alpha \in[-1,1], R_{\alpha} h(K, \cdot)$ is again a support function of some convex body $K_{\alpha}$ and that $K \mapsto K_{\alpha}$ is a Minkowski-endomorphism. Moreover, any $\Phi \in \operatorname{End}_{\mathrm{M}}\left(\mathcal{K}^{d}\right)$ is a mixture of the prototypes $K \mapsto K_{\alpha}$, where mixing (of the corresponding support functions) is now understood with respect to a distribution (generalized function) $\tilde{F}$ on $[-1,1]$. As convexity controls derivatives up to order 2 , we can show that $\tilde{F}$ must be an element of a certain subclass $\mathcal{E}_{2}^{\prime}[-1,1]$ of distributions of order at most $2 . \mathcal{E}_{2}^{\prime}[-1,1]$ is given explicitly by (2.17).

Theorem 1.3. Assume $d \geq 3$. For every $\Phi \in \operatorname{End}_{\mathrm{M}}\left(\mathcal{K}^{d}\right)$ there exists a distribution $\tilde{F} \in \mathcal{E}_{2}^{\prime}[-1,1]$ such that

$$
h(\Phi K, \cdot)=\tilde{F}_{(\alpha)}\left(R_{\alpha} h(K, \cdot)\right)
$$

holds for all $K \in \mathcal{K}^{d}$. $\tilde{F}$ is uniquely determined by (1.2) and will be called the mixing distribution of $\Phi$. Furthermore,

i) $\Phi$ is symmetric $\Longleftrightarrow \tilde{F}$ is even,

ii) $\Phi$ is uniformly continuous $\Longleftrightarrow \tilde{F}$ is a finite signed measure on $[-1,1]$,

iii) $\Phi$ is monotonic $\Longleftrightarrow \tilde{F}$ is a finite positive measure on $[-1,1]$.

The notation $\tilde{F}_{(\alpha)}$ indicates that the distribution $\tilde{F}$ acts on the variable $\alpha$. If $\alpha \mapsto R_{\alpha} h(K, \cdot)$ is not sufficiently smooth, (1.2) must be interpreted in the sense of distributions.

Theorem 1.3 identifies the set $\operatorname{End}_{M}\left(\mathcal{K}^{d}\right)$ with a certain class of distributions. It does not give a complete characterization: Not every distribution in $\mathcal{E}_{2}^{\prime}[-1,1]$ induces a Minkowski-endomorphism. In the case of weakly monotonic Minkowskiendomorphisms, however, such a characterization is possible. To formulate this characterization, we will need the notion of a linear measure on $[-1,1]$, which is, by definition, any multiple of the measure

$$
\int_{(\cdot)} \alpha\left(1-\alpha^{2}\right)^{(d-3) / 2} d \alpha
$$

Linear measures play a special role: If a linear measure is interpreted as a distribution $\tilde{F}$, it is the mixing distribution of the Minkowski-endomorphism $K \mapsto\{a \cdot s(K)\}$ for some $a \in \mathbb{R}$.

Theorem 1.4. Assume $d \geq 3$. The mixing distribution of a weakly monotonic Minkowski-endomorphism is a finite signed measure. It is positive up to addition of a linear measure.

Conversely, if $\tilde{\mu}$ is a finite signed measure on $[-1,1]$, which is positive up to addition of a linear measure, then

$$
\int_{-1}^{1} R_{\alpha} h(K, \cdot) d \tilde{\mu}(\alpha)
$$

is the support function of a convex body $K^{\prime}$ and the mapping $\Phi: K \mapsto K^{\prime}$ is a weakly monotonic Minkowski-endomorphism on $\mathcal{K}^{d}$.

Theorem 1.4 shows in particular that for every weakly monotonic Minkowski-endomorphism $\Phi$ there is a constant $a \in \mathbb{R}$, such that $\Phi+a \cdot s$ is monotonic. 
1.2. Blaschke-endomorphisms. Besides the Minkowski-addition of convex bodies, the so-called Blaschke-addition has been considered in the literature. We restrict the latter addition to the class

$$
\mathcal{K}_{0}^{d}:=\left\{K \in \mathcal{K}^{d} \mid \operatorname{int} K \neq \emptyset, s(K)=o\right\}
$$

of all convex bodies with non-empty interior and Steiner point at the origin. Let $S(K, \cdot)$ be the usual surface area measure of $K$ (of order $d-1$ on $S^{d-1}$ ). For $K, M \in$ $\mathcal{K}_{0}^{d}$ Minkowski's existence theorem (see Section 2.3) guarantees that $S(K, \cdot)+$ $S(M, \cdot)$ is the surface area measure of a uniquely determined convex body $K \# M \in$ $\mathcal{K}_{0}^{d}$, the Blaschke-sum of $K$ and $M$. In analogy with the notion of Minkowskiadditivity, we call a mapping $\Psi: \mathcal{K}_{0}^{d} \rightarrow \mathcal{K}_{0}^{d}$ Blaschke-additive if it satisfies

$$
\Psi(K \# M)=\Psi K \# \Psi M, \quad K, M \in \mathcal{K}_{0}^{d} .
$$

Definition 1.5. Any continuous, Blaschke-additive and $S O_{d^{-}}$equivariant mapping from $\mathcal{K}_{0}^{d}$ into itself is called a Blaschke-endomorphism.

The (abstract) convex cone of all Blaschke-endomorphisms is denoted by $\operatorname{End}_{\mathrm{B}}\left(\mathcal{K}_{0}^{d}\right)$.

Example 1.6. For $K \in \mathcal{K}_{0}^{d}$ and $L \in \mathcal{L}_{k}^{d}, k \in\{2, \ldots, d-1\}$, let $B_{L}(K)$ be the Blaschke section body relative to $L . B_{L}(K)$ is the invariant mean (in the sense of Blaschke addition) of all intersections of $K$ with translates of $L$ :

$$
S_{L}\left(B_{L}(K), \cdot\right)=\int_{L^{\perp}} S_{L}((K+x) \cap L, \cdot) d \lambda_{d-k}(x) .
$$

Here $\lambda_{d-k}$ denotes Lebesgue-measure on the orthogonal space $L^{\perp}$ of $L$. $(K+x) \cap L$ is a convex body in $L$, and $S_{L}((K+x) \cap L, \cdot)$ denotes its surface area measure (of order $k-1$ ) relative to $L$. Again, Minkowski's existence theorem (applied in $L$ ) guarantees the existence of a unique convex body $B_{L}(K)$ with Steiner point at the origin. WeIL 23 defined the Blaschke section body $B_{k}(K) \in \mathcal{K}_{0}^{d}$ as the invariant mean of $B_{L}(K)$ with respect to $\nu_{k}$ :

$$
S\left(B_{k}(K), \cdot\right)=\int_{\mathcal{L}_{k}^{d}} S_{L}\left(B_{L}(K), \cdot\right) \nu_{k}(d L),
$$

where the measure $S_{L}\left(B_{L}(K), \cdot\right)$ is interpreted as a measure on $S^{d-1}$ with support in $S^{d-1} \cap L$. With certain modifications, the above definition can be extended to include the case of line intersections $(k=1)$; see Goodey ET AL. [9]. This note also shows that the mapping $K \mapsto B_{k}(K)$ is Blaschke-additive and continuous. Hence, we have $B_{k} \in \operatorname{End}_{\mathrm{B}}\left(\mathcal{K}_{0}^{d}\right), k=1, \ldots, d-1$.

For $d=2$, we have

$$
K \# M=K+M, \quad K, M \in \mathcal{K}_{0}^{2} .
$$

Therefore, Blaschke-endomorphisms in $\mathbb{R}^{2}$ can be written as "mixtures" of the prototypes $K \mapsto \vartheta K, \vartheta \in S O_{2}$, due to the corresponding result for Minkowskiendomorphisms; see Corollary 2.8, In contrast to the case of Minkowski-endomorphisms, we can state a complete characterization for Blaschke-endomorphisms in higher dimensions. This result again uses the generalized spherical Radon transform and the fact that this transform can naturally be extended to measures (see (2.20) for a formal definition of this extension). 
Theorem 1.7. Assume $d \geq 3$. For $\Psi \in \operatorname{End}_{\mathrm{B}}\left(\mathcal{K}_{0}^{d}\right)$ there exists a finite signed measure $\tilde{\mu}$ on $[-1,1]$ with the property that the sum of $\tilde{\mu}$ and a suitable linear measure is positive and non-zero, such that

$$
S(\Psi K, \cdot)=\int_{-1}^{1} R_{\alpha}(S(K, \cdot)) d \tilde{\mu}(\alpha), \quad K \in \mathcal{K}_{0}^{d} .
$$

$\tilde{\mu}$ is determined by (1.7) up to addition of a linear measure.

Conversely, let $\tilde{\mu}$ be a signed measure on $[-1,1]$. If the sum of $\tilde{\mu}$ and a suitable linear measure is positive and non-zero, then the right-hand side of (1.7) is the surface area measure of some convex body $K^{\prime} \in \mathcal{K}_{0}^{d}$ and the mapping $K \mapsto K^{\prime}$ is a Blaschke-endomorphism.

Any measure $\tilde{\mu}$ that satisfies (1.7) is called a mixing measure of $\Psi$.

1.3. Adjoint endomorphisms and applications. The class of Blaschke-endomorphisms essentially coincides with a subclass of $\operatorname{End}_{\mathrm{M}}\left(\mathcal{K}^{d}\right)$ by application of an adjoining operator. Let $\operatorname{End}_{\mathrm{M}}^{*}\left(\mathcal{K}^{d}\right)$ be the class of all $\Phi \in \operatorname{End}_{\mathrm{M}}\left(\mathcal{K}^{d}\right)$ which satisfy

i) $\Phi$ is weakly monotonic,

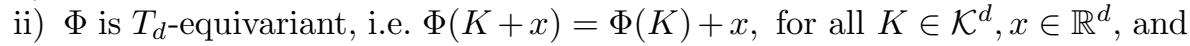

iii) $\Phi$ is non-degenerate, i.e. there is a $K \in \mathcal{K}^{d}$ such that $\Phi K$ is not a singleton. Note that $\operatorname{End}_{\mathrm{M}}^{*}\left(\mathcal{K}^{d}\right)$ is essentially the class of endomorphisms considered by Schneider which are in addition weakly monotonic. In the following, we make use of the special mixed volume

$$
V(K, M[d-1]):=\frac{1}{d} \int_{S^{d-1}} h(K, u) d S(M, u)
$$

of the convex bodies $K, M \in \mathcal{K}^{d}$.

Theorem 1.8. Assume $d \geq 3$. For any $\Phi \in \operatorname{End}_{\mathrm{M}}^{*}\left(\mathcal{K}^{d}\right)$ there is a uniquely determined Blaschke-endomorphism $\Phi^{*}$ such that

$$
V(\Phi K, M[d-1])=V\left(K,\left(\Phi^{*} M\right)[d-1]\right)
$$

holds for all $K \in \mathcal{K}^{d}$ and $M \in \mathcal{K}_{0}^{d}$.

The mapping $\Lambda: \operatorname{End}_{\mathrm{M}}^{*}\left(\mathcal{K}^{d}\right) \rightarrow \operatorname{End}_{\mathrm{B}}\left(\mathcal{K}_{0}^{d}\right), \Phi \mapsto \Phi^{*}$, has the following properties:

i) $\Lambda$ is additive: $(\Phi+\chi)^{*}=\Phi^{*} \# \chi^{*}$, for all $\Phi, \chi \in \operatorname{End}_{\mathrm{M}}^{*}\left(\mathcal{K}^{d}\right)$,

ii) $\Lambda$ is positive homogeneous of degree $1 /(d-1)$,

iii) $\Lambda$ is a bijection, and the mixing measure of $\Phi$ is a mixing measure of $\Phi^{*}$,

iv) $\Lambda$ preserves injectivity: $\Phi$ is injective if and only if $\Phi^{*}$ is injective.

Example 1.9. Fix $2 \leq k \leq d-1$. The Minkowski-endomorphism $P_{k}$ is not $T_{d^{-}}$ equivariant. Define

$$
Q_{k}(K):=P_{k}(K)-s\left(P_{k}(K)\right)+s(K), \quad K \in \mathcal{K}^{d} .
$$

Then $Q_{k}(K)$ and $P_{k}(K)$ are equal up to translation and $Q_{k} \in \operatorname{End}_{\mathrm{M}}^{*}\left(\mathcal{K}^{d}\right)$. Furthermore, $Q_{k}$ is injective if and only if $P_{k}$ is. In GoODEY ET AL. 9] the relation $Q_{k}^{*}=B_{k}$ is shown and used to transfer known injectivity results from $P_{k}$ to $B_{k}$.

SCHNEIDER [17] has shown that the only convex bodies $K$ that solve the "eigenvalue problem"

$$
P_{d-1}(K)=\beta K
$$

for some real $\beta$ are (possibly degenerate) balls. If $K \neq\{o\}, \beta$ is uniquely determined. Goodey [6] showed that this is even true if $P_{d-1}$ is replaced by $P_{k}$ for 
arbitrary $1 \leq k \leq d-1$. This result can be extended to all weakly monotonic Minkowski-endomorphisms if trivial cases are excluded. $\Phi \in \operatorname{End}_{\mathrm{M}}\left(\mathcal{K}^{d}\right)$ is called trivial if there are $\tau_{1}, \tau_{2} \geq 0$ and $\lambda \in \mathbb{R}$ such that

$$
\Phi K=\tau_{1} K+\tau_{2}(-K)+\lambda s(K)
$$

for all $K \in \mathcal{K}^{d}$. Let $B^{d}$ be the unit ball in $\mathbb{R}^{d}$ and $\varpi_{d}$ its surface area.

Theorem 1.10. Assume $d \geq 3$ and let $\Phi \in \operatorname{End}_{\mathrm{M}}\left(\mathcal{K}^{d}\right)$ be weakly monotonic and non-trivial. If $K \in \mathcal{K}^{d}$ satisfies

$$
\Phi K=\beta K+x
$$

for some $\beta \in \mathbb{R}$ and $x \in \mathbb{R}^{d}$, then $K$ is a (possibly degenerate) ball. If $K$ is not a singleton, then $\beta$ is the radius of the ball $\Phi B^{d}$.

The final result of this note shows that Theorem 1.10 transfers to Blaschkeendomorphisms. A Blaschke-endomorphism $\Psi$ is called trivial if there are $\tau_{1}, \tau_{2} \geq 0$ such that

for all $K \in \mathcal{K}_{0}^{d}$.

$$
\Psi K=\tau_{1} K+\tau_{2}(-K)
$$

Theorem 1.11. Assume $d \geq 3$ and let $\Psi \in \operatorname{End}_{\mathrm{B}}\left(\mathcal{K}_{0}^{d}\right)$ be non-trivial. If $K \in \mathcal{K}_{0}^{d}$ satisfies

$$
\Psi K=\beta K
$$

for some $\beta \in \mathbb{R}$, then $K$ is a ball and $\beta$ is the radius of the ball $\Psi B^{d}$.

In Section 2, we will present the tools required to prove these results. The proofs will be given in Section 3 .

\section{KNOWN RESULTS AND TOOLS}

2.1. Distributions on the sphere. The application of distributions to solve convex geometric problems is not new: BERG [1, used them as a tool to characterize the first surface area measures of convex bodies. They also play an important role when extending results from the class of zonoids to general centrally symmetric convex bodies; see the survey 7 and, more recently, 4]. In the following, we recall known results that will be needed later and complete them, where necessary.

Distributions on the sphere are special cases of distributions on differentiable manifolds; see e.g. Schwartz [20, pp. 31-33]. Distributions on the sphere are continuous linear functionals on the locally convex space of infinitely differentiable functions on $S^{d-1}$. Differentiability of a function $\varphi: S^{d-1} \rightarrow \mathbb{R}$ corresponds to differentiability of its positive homogeneous extension of degree 0 on $\mathbb{R}^{d} \backslash\{o\}$. Let $\mathcal{D}_{k}:=\mathcal{D}_{k}\left(S^{d-1}\right), k \in \mathbb{N}_{0}$, be the vector space of all $k$-times continuously differentiable real functions $\varphi$ on $S^{d-1}$ endowed with the usual norm

$$
\|\varphi\|_{\mathcal{D}_{k}}:=\max \left\{\left|\partial^{q} \varphi(u)\right|\left|u \in S^{d-1},\right| q \mid \leq k\right\} .
$$

Here, $q=\left(q_{1}, \ldots, q_{d}\right) \in \mathbb{N}_{0}^{d}$ is a multi-index with $|q|:=q_{1}+\ldots+q_{d} \leq k$ and

$$
\partial^{q} \varphi(u):=\frac{\partial^{|q|}}{\partial x_{1}^{q_{1}} \cdots \partial x_{d}^{q_{d}}} \varphi(u) .
$$

$\mathcal{D}_{k}$ is a Banach space and $\mathcal{D}_{0}=\mathcal{C}\left(S^{d-1}\right)=: \mathcal{C}$.

We equip $\mathcal{D}:=\bigcap_{k=0}^{\infty} \mathcal{D}_{k}$ with the projective topology. $\mathcal{D}$ is a Fréchet space. A sequence of functions $\varphi_{n} \in \mathcal{D}$ converges in $\mathcal{D}$ to a function $\varphi$, if and only if the 
functions $\partial^{q} \varphi_{n}$ converge uniformly on $S^{d-1}$ to $\partial^{q} \varphi$ for all multi-indices $q$. In WEIL 22], derivatives of spherical functions are understood with respect to their positive homogeneous extension of degree one. This approach leads to the same classes $\mathcal{D}_{k}$ with equivalent norms. We mark the dual space by a prime and call the elements of $\mathcal{D}^{\prime}$ distributions on the sphere. The elements of $\mathcal{D}_{k}^{\prime}$ are the distributions of order at most $k$. Due to the compactness of $S^{d-1}$ we have

$$
\mathcal{D}^{\prime}=\bigcup_{k=0}^{\infty} \mathcal{D}_{k}^{\prime}
$$

so all spherical distributions are of finite order. $\mathcal{D}^{\prime}$ will be endowed with the usual weak topology.

Let $\mathcal{M}$ be the vector space of finite signed measures on the Borel sets of the unit sphere (with the weak topology). The Riesz representation theorem implies

$$
\mathcal{C}^{\prime}=\mathcal{D}_{0}^{\prime}=\mathcal{M}
$$

where every signed measure $\mu$ is canonically identified with the distribution

$$
\varphi \mapsto \mu(\varphi):=\int_{S^{d-1}} \varphi d \mu, \quad \varphi \in \mathcal{D} .
$$

$F \in \mathcal{D}^{\prime}$ is called regular if it can be represented in the form

$$
F(\varphi)=\int_{S^{d-1}} \varphi(u) f(u) d \omega_{d-1}(u), \quad \varphi \in \mathcal{D}
$$

with some integrable function $f$ on $S^{d-1}$. Here and in the following, $\omega_{k-1}$ denotes the spherical Lebesgue-measure on the unit sphere $S^{k-1}$ of $\mathbb{R}^{k}$. Its total mass is

$$
\varpi_{k}=\omega_{k-1}\left(S^{k-1}\right)=\frac{2 \pi^{k / 2}}{\Gamma\left(\frac{k}{2}\right)} .
$$

Conversely, by (2.1), any integrable function $f$ can be interpreted as a (regular) distribution, or, equivalently, as a measure having the (signed) density $f$. As the integrals in (2.1) determine $f$ up to a set of measure zero, we can identify $f$ with $F$.

The Laplace-Beltrami operator $\Delta_{d}$ on $S^{d-1}$, given by

$$
\Delta_{d}=\frac{\partial^{2}}{\partial x_{1}^{2}}+\ldots+\frac{\partial^{2}}{\partial x_{d}^{2}}
$$

is a distribution of order two. Although we will only use distributions of order at most two, most of the results in this section are given for $\mathcal{D}^{\prime}$, as this more general setting requires no further effort.

If $f$ is a real-valued function on $S^{d-1}$ and $\vartheta \in S O_{d}$, we define

$$
(\vartheta f)(u):=f\left(\vartheta^{-1} u\right), \quad u \in S^{d-1} .
$$

This induces an action of the rotation group $S O_{d}$ on the function spaces $\mathcal{D}_{k}$. It is easy to see that this operation is continuous on $\mathcal{D}_{k}, k \in \mathbb{N}_{0}$ (i.e. the mapping $S O_{d} \times \mathcal{D}_{k} \rightarrow \mathcal{D}_{k},(\vartheta, f) \mapsto \vartheta f$ is continuous $)$. Hence it is also continuous on $\mathcal{D}$. $S O_{d}$ operates continuously on $\mathcal{D}^{\prime}$ by

$$
(\vartheta F)(\varphi):=F\left(\vartheta^{-1} \varphi\right), \quad \varphi \in \mathcal{D}
$$

for distributions $F$ and $\vartheta \in S O_{d}$. If $F=\mu \in \mathcal{D}$ is a signed measure, $\vartheta \mu$ is the image measure of $\mu$ under $\vartheta$. 
For the following, let $p \in S^{d-1}$ be a fixed point, the "north pole" of the unit sphere. $S O_{p^{\perp}} \subset S O_{d}$ denotes the subgroup of all rotations fixing $p$. Let $\nu_{p^{\perp}}$ be the normalized Haar-measure on this subgroup. Details on invariant measures, with emphasis on integral geometric applictaions, can be found in SCHNEIDER AND WeIL [19]. A distribution $F \in \mathcal{C}$ is called zonal (with respect to $p$ ), if it is invariant with respect to all rotations fixing $p$, i.e. if $\vartheta F=F$ holds for all $\vartheta \in S O_{p^{\perp}}$. For any function $f \in \mathcal{C}$ its rotational symmetrization $\bar{f}$ (with respect to $p$ ) is given by

$$
\bar{f}(u):=\int_{S O_{p} \perp}(\vartheta f)(u) \nu_{p^{\perp}}(d \vartheta), \quad u \in S^{d-1} .
$$

We call the function $g$ a finite rotation mean of $f$ about $p$ if there are $m \in \mathbb{N}$ and $\vartheta_{1}, \ldots, \vartheta_{m} \in S O_{p^{\perp}}$ such that $g$ is a convex combination of the functions $\vartheta_{1} f, \ldots, \vartheta_{m} f$.

Lemma 2.1. For fixed $k \in \mathbb{N}_{0}$, the mapping $\varphi \mapsto \bar{\varphi}$ is a continuous endomorphism of $\mathcal{D}_{k}$. For any $\varphi \in \mathcal{D}_{k}$ there exists a sequence of finite rotation means of $\varphi$ about $p$ that converges to $\bar{\varphi}$ in $\mathcal{D}_{k}$.

In addition, all statements are true with $\mathcal{D}_{k}$ replaced by $\mathcal{D}$.

Proof. $\bar{\varphi}$ has at least the same order of differentiability as $\varphi$. The linearity of $\varphi \mapsto \bar{\varphi}$ is clear, and its continuity follows from the continuity of the operation of $S O_{d}$ on $\mathcal{D}_{k}$.

We will construct a sequence $\left(\sigma_{m}\right)$ of probability measures with finite support on $S O_{p^{\perp}}$, for which

$$
\int_{S O_{p^{\perp}}}(\vartheta \varphi) \sigma_{m}(d \vartheta) \rightarrow \int_{S O_{p^{\perp}}}(\vartheta \varphi) \nu_{p^{\perp}}(d \vartheta)=\bar{\varphi}, \quad m \rightarrow \infty,
$$

holds for all $\varphi \in \mathcal{D}_{k}$. Here, convergence is understood in $\mathcal{D}_{k}$. The left-hand side of (2.2) is a finite rotation mean of $\varphi$ about $p$.

For any $m \in \mathbb{N}$, there is a partition of the compact set $S O_{p^{\perp}}$ into measurable sets $B_{1}, \ldots, B_{k_{m}}$ of diameter at most $1 / \mathrm{m}$. (Any metric that induces the topology on $S O_{p^{\perp}}$ can be used here.) Define the probability measure

$$
\sigma_{m}:=\sum_{i=1}^{k_{m}} \nu_{p^{\perp}}\left(B_{i}\right) \delta_{\vartheta_{i}},
$$

where $\vartheta_{1} \in B_{1}, \ldots, \vartheta_{k_{m}} \in B_{k_{m}}$ are arbitrary and $\delta_{\vartheta}$ denotes the probability measure supported by $\{\vartheta\}$. Assume $\varphi \in \mathcal{D}_{k}$ and let $q$ be a multi-index with $|q| \leq k$. We have

$$
\begin{aligned}
\left\|\boldsymbol{\partial}^{q} \int_{S O_{p^{\perp}}}(\vartheta \varphi) \sigma_{m}(d \vartheta)-\boldsymbol{\partial}^{q} \bar{\varphi}\right\|_{\infty} & =\left\|\sum_{i=1}^{k_{m}} \int_{B_{i}} \partial^{q}\left(\vartheta_{i} \varphi-\vartheta \varphi\right) \nu_{p^{\perp}}(d \vartheta)\right\|_{\infty} \\
& \leq \sum_{i=1}^{k_{m}} \int_{B_{i}}\left\|\partial^{q}\left(\vartheta_{i} \varphi-\vartheta \varphi\right)\right\|_{\infty} \nu_{p^{\perp}}(d \vartheta) .
\end{aligned}
$$

The continuous mapping $S O_{p^{\perp}} \rightarrow \mathcal{C}, \vartheta \mapsto \partial^{q}(\vartheta \varphi)$ is uniformly continuous on the compact group $S O_{p^{\perp}}$. Hence, the last expression converges to zero, as $m \rightarrow \infty$.

The remaining assertion for $\mathcal{D}$ follows from the fact that the above constructed sequence converges in $\mathcal{D}$ if $\varphi \in \mathcal{D}$ is assumed. 
We will work with Fourier expansions (spherical harmonic expansions) of distributions. Details about spherical harmonics can be found e.g. in Groemer's book [5. Let $\mathcal{H}_{n}^{d} \subset \mathcal{C}$ be the (finite-dimensional) vector space of spherical harmonics on $S^{d-1}$ of degree $n \in \mathbb{N}_{0}$. There is a uniquely determined zonal function $f \in \mathcal{H}_{n}^{d}$ with $f(p)=1$ :

$$
f(u)=P_{n}^{d}(\langle p, u\rangle), \quad u \in S^{d-1} .
$$

Here, $\langle\cdot, \cdot\rangle$ is the usual scalar product on $\mathbb{R}^{d}$ and $P_{n}^{d}$ is the Legendre polynomial of dimension $d$ and degree $n$. The Legendre-polynomials $P_{0}^{d}, P_{1}^{d}, P_{2}^{d}, \ldots$ form an orthogonal system of functions on $[-1,1]$ with respect to the measure $\tilde{\mu}_{d}$ given by

$$
d \tilde{\mu}_{d}:=\varpi_{d-1}\left(1-\alpha^{2}\right)^{(d-3) / 2} d \alpha .
$$

Therefore, $P_{n}^{d}$ is a constant multiple of the Gegenbauer polynomial of upper index $\nu=(d-2) / 2$ and degree $n$. Later we will need a strengthened version of the well-known fact

$$
\left|P_{n}^{d}(\alpha)\right| \leq 1, \quad-1 \leq \alpha \leq 1
$$

$n \geq 0, d \geq 2$.

Lemma 2.2. We have for $n \geq 1$ and $d \geq 3$

$$
\left|P_{n}^{d}(\alpha)\right|<1, \quad-1<\alpha<1 .
$$

Proof. (2.5) follows from the explicit integral representation

$$
P_{n}^{d}(\alpha)=c_{n, d} \int_{0}^{\pi}\left(\alpha+i\left(1-\alpha^{2}\right)^{1 / 2} \cos \gamma\right)^{n}(\sin \gamma)^{d-3} d \gamma
$$

where $c_{n, d} \in \mathbb{R}$ is such that the right-hand side is 1 for $\alpha=1$; see [3, formula (31) on p. 177].

We write $f \sim \sum_{n=0}^{\infty} f_{n}$ with

$$
f_{n}:=\frac{\operatorname{dim} \mathcal{H}_{n}^{d}}{\varpi_{d}} \int_{S^{d-1}} f(u) P_{n}^{d}(\langle u, \cdot\rangle) d \omega_{d-1}(u) \in \mathcal{H}_{n}^{d}
$$

for the Fourier expansion of $f \in \mathcal{C}$. It converges in the $L_{2}$ sense to $f$. If $f \in \mathcal{D}$, the Fourier expansion converges to $f$ in $\mathcal{D}$. Fourier expansions can also be defined for distributions $F \in \mathcal{D}^{\prime}$. Extending (2.6), we define the regular distribution $F_{n} \in \mathcal{H}_{n}^{d}$ by

$$
F_{n}:=\frac{\operatorname{dim} \mathcal{H}_{n}^{d}}{\varpi_{d}} F_{(u)}\left(P_{n}^{d}(\langle u, \cdot\rangle)\right)
$$

and write $F \sim \sum_{n=0}^{\infty} F_{n}$. We have $F_{n}(\varphi)=F\left(\varphi_{n}\right)$. Together with the completeness of $\mathcal{D}^{\prime}$ (any sequence of distributions with pointwise limits converges in $\mathcal{D}^{\prime}$ to a distribution), this implies $\lim _{m \rightarrow \infty} \sum_{n=0}^{m} F_{n}=F$ in $\mathcal{D}^{\prime}$.

If $F=\mu$ is a measure, $\mu_{1}(\cdot)=d \varpi_{d}^{-1}\langle c(\mu), \cdot\rangle$, where

$$
c(\mu):=\int_{S^{d-1}} u d \mu(u) \in \mathbb{R}^{d}
$$

is the centroid of $\mu$. 
2.2. Rotation commuting operators on spaces of spherical functions. In this section we gather properties of rotation commuting linear and continuous selfmappings of $\mathcal{D}, \mathcal{C}, \mathcal{M}$ and $\mathcal{D}^{\prime}$. An operator $A$ commutes with rotations if $\vartheta \circ A=A \circ \vartheta$ holds for all $\vartheta \in S O_{d}$. The vector spaces of these operators will be called $\operatorname{End}(\mathcal{D})$, $\operatorname{End}(\mathcal{C}), \operatorname{End}(\mathcal{M})$ and $\operatorname{End}\left(\mathcal{D}^{\prime}\right)$, respectively.

The most important example of such an operator for our purposes is the generalized spherical Radon transform $R_{\alpha}$ with parameter $\alpha \in[-1,1]$. For $f \in \mathcal{C}$, we define

$$
\left(R_{\alpha} f\right)(u)=\varpi_{d-1}^{-1} \int_{S\left(u^{\perp}\right)} f\left(\alpha u+\sqrt{1-\alpha^{2}} v\right) d \omega_{d-2}(v), \quad u \in S^{d-1} .
$$

Here $S(L)=S^{d-1} \cap L$ denotes the unit sphere of the linear subspace $L \subset \mathbb{R}^{d}$. As mentioned above, $\left(R_{\alpha} f\right)(u)$ is the average of the $f$-values on the subsphere of all unit vectors in the hyperplane with normal $u$ and (signed) distance $\alpha$ from the origin. $R_{0}$ is the usual spherical Radon transform (sometimes called MinkowskiFunk transform) which gives the averages of $f$ on great circles. $R_{1}$ is the identity, and $R_{-1}$ is the reflection at the origin. We have

$$
\left(R_{\langle p, u\rangle} f\right)(p)=\bar{f}(u), \quad u \in S^{d-1} .
$$

Fix $\alpha \in[-1,1]$. BERG [1, p. 42] has shown that $R_{\alpha} \in \operatorname{End}(\mathcal{C})$ and that this operator is self-adjoint if $\mathcal{C}$ is endowed with the canonical inner product of $L_{2}-$ functions:

$$
\int_{S^{d-1}}\left(R_{\alpha} f\right)(u) g(u) d \omega_{d-1}(u)=\int_{S^{d-1}} f(u)\left(R_{\alpha} g\right)(u) d \omega_{d-1}(u), \quad f, g \in \mathcal{C} .
$$

Next, we will show that $\frac{\partial^{k}}{\partial \alpha^{k}} R_{\alpha}$ is a continuous linear mapping from $\mathcal{D}$ into itself, which can be extended continuously to a mapping in $\operatorname{End}\left(\mathcal{D}^{\prime}\right)$. It is convenient to use spherical harmonics in this context. SCHNEIDER [14] showed that $R_{\alpha}$ acts as a multiple of the identity on $\mathcal{H}_{n}^{d}$ :

$$
R_{\alpha} f_{n}=P_{n}^{d}(\alpha) f_{n}
$$

for all $f_{n} \in \mathcal{H}_{n}^{d}$. In the following, derivatives of functions on $[-1,1]$ are understood to be one-sided if evaluated at one of the endpoints of this interval.

\section{Lemma 2.3.}

1) For $\varphi \in \mathcal{D}, u \in S^{d-1}$, the function $\alpha \mapsto\left(R_{\alpha} \varphi\right)(u)$ is infinitely differentiable on $[-1,1]$,

2) for all $k \in \mathbb{N}_{0}$, the operator given by

$$
\left(\frac{\partial^{k}}{\partial \alpha^{k}} R_{\alpha}(\varphi)\right)(u):=\frac{\partial^{k}}{\partial \alpha^{k}}\left(\left(R_{\alpha} \varphi\right)(u)\right), \quad \varphi \in \mathcal{D}, u \in S^{d-1}
$$

satisfies $\frac{\partial^{k}}{\partial \alpha^{k}} R_{\alpha} \in \operatorname{End}(\mathcal{D})$

3) there is a unique continuous extension of the operator in 2) to an operator in $\operatorname{End}\left(\mathcal{D}^{\prime}\right)$, given by

$$
\left(\frac{\partial^{k}}{\partial \alpha^{k}} R_{\alpha}(F)\right)(\varphi)=F\left(\frac{\partial^{k}}{\partial \alpha^{k}} R_{\alpha}(\varphi)\right), \quad F \in \mathcal{D}^{\prime}, \varphi \in \mathcal{D},
$$

4) if the operator $R_{\alpha}$ in 3) (with $k=0$ ) is restricted to $\mathcal{M}$, we have $R_{\alpha} \in$ $\operatorname{End}(\mathcal{M})$ 
Proof. Let $\varphi \sim \sum_{n=0}^{\infty} \varphi_{n}$ be the Fourier expansion of $\varphi \in \mathcal{D}$ and let $q$ be an arbitrary multi-index. Equation (2.11) implies

$$
\sum_{k=0}^{\infty} \partial^{q} \frac{\partial^{k}}{\partial \alpha^{k}}\left(R_{\alpha} \varphi_{n}\right)=\sum_{k=0}^{\infty}\left(\frac{d^{k}}{d \alpha^{k}} P_{n}^{d}(\alpha)\right) \partial^{q} \varphi_{n}
$$

A combination of [5, equation (3.3.22)] and (2.4) gives

$$
\left|\frac{d^{k}}{d \alpha^{k}} P_{n}^{d}(\alpha)\right| \leq c_{1} \cdot n^{2 k}
$$

for some constant $c_{1}>0$ depending only on $d$. Furthermore, there is a constant $c_{2}=c_{2}(|q|, d)$ such that

$$
\left\|\partial^{q} \varphi_{n}\right\|_{\infty} \leq c_{2} \cdot n^{|q|+d / 2-1-m}\left\|\Delta_{d}^{m / 2} \varphi\right\|_{\infty}
$$

for all $m \in 2 \mathbb{N}$. This follows from SEELEy [13, Theorem 4(b)], with an argument given in [18, p. 186]. If we put $m=2(\lceil k+|q| / 2+d / 4+1\rceil)$, it follows that the series in (2.13) converges absolutely (in the maximum norm). Hence, the derivatives

$$
\partial^{q} \frac{\partial^{k}}{\partial \alpha^{k}}\left(R_{\alpha} \varphi\right)=\sum_{n=0}^{\infty} \partial^{q} \frac{\partial^{k}}{\partial \alpha^{k}}\left(R_{\alpha} \varphi_{n}\right)
$$

exist and

$$
\left\|\frac{\partial^{k}}{\partial \alpha^{k}}\left(R_{\alpha} \varphi\right)\right\|_{\mathcal{D}_{|q|}} \leq c_{3}\|\varphi\|_{\mathcal{D}_{m}}
$$

for some $c_{3}=c_{3}(k,|q|, d)$. This shows assertions 1) and 2), as $\frac{\partial^{k}}{\partial \alpha^{k}} R_{\alpha}$ is obviously a linear operator that commutes with rotations.

To show 3 ) we abbreviate the operator defined in (2.12) by $A$. So, $A$ is a mapping from $\mathcal{D}^{\prime}$ into itself that satisfies

$$
(A F)(\varphi)=F\left(\frac{\partial^{k}}{\partial \alpha^{k}} R_{\alpha}(\varphi)\right), \quad F \in \mathcal{D}^{\prime}, \varphi \in \mathcal{D} .
$$

It is well defined due to 2), and we obviously have $A \in \operatorname{End}\left(\mathcal{D}^{\prime}\right)$. From (2.10) and the fact that integration and differentiation can be interchanged, we get

$$
\begin{aligned}
\int_{S^{d-1}} & \left(\frac{\partial^{k}}{\partial \alpha^{k}} R_{\alpha}(\psi)\right)(u) \varphi(u) d \omega_{d-1}(u) \\
= & \int_{S^{d-1}} \psi(u)\left(\frac{\partial^{k}}{\partial \alpha^{k}} R_{\alpha}(\varphi)\right)(u) d \omega_{d-1}(u), \quad \psi, \varphi \in \mathcal{D} .
\end{aligned}
$$

Hence, for the regular distribution $F=\psi$ we have $A \psi=A F=\frac{\partial^{k}}{\partial \alpha^{k}} R_{\alpha} \psi$. In other words, $A$ coincides with $\frac{\partial^{k}}{\partial \alpha^{k}} R_{\alpha}$ on $\mathcal{D}$. As $\mathcal{D}$ is a dense subset of $\mathcal{D}^{\prime}, A$ is the unique continuous extension of $\frac{\partial^{k}}{\partial \alpha^{k}} R_{\alpha}$ to $\mathcal{D}^{\prime}$.

4 ) is evident, as for any signed measure $\mu$, the mapping

$$
\varphi \mapsto\left(R_{\alpha} \mu\right)(\varphi)=\int_{S^{d-1}}\left(R_{\alpha} \varphi\right)(u) d \mu(u)
$$

is a distribution of order zero, i.e. a signed measure. 
The material collected so far enables us to give characterization theorems for rotation-commuting continuous linear operators on function spaces on the sphere. It turns out that these operators are "mixtures" of the prototypes $\frac{\partial^{k}}{\partial \alpha^{k}} R_{\alpha}$. Before stating the general result consider the case $k=0$ and let $\mathcal{M}[-1,1]$ be the vector space of finite signed Borel measures on $[-1,1]$, endowed with the weak topology. For any $\tilde{\mu} \in \mathcal{M}[-1,1]$, the mapping

$$
A_{\tilde{\mu}}: f \mapsto \int_{-1}^{1} R_{\alpha} f d \tilde{\mu}(\alpha), \quad f \in \mathcal{C},
$$

is an element of $\operatorname{End}(\mathcal{C})$. The next proposition follows from DunkL [2, Theorem 8].

Proposition 2.4. The mapping $\Theta: \tilde{\mu} \mapsto A_{\tilde{\mu}}$, where $A_{\tilde{\mu}}$ is given by (2.15), is an isomorphism from $\mathcal{M}[-1,1]$ onto $\operatorname{End}(\mathcal{C})$.

$\Theta^{-1}(A)$ will be called the mixing measure of $A \in \operatorname{End}(\mathcal{C})$. The class of all $A \in \operatorname{End}(\mathcal{C})$ which are monotonic $(f \geq 0 \Rightarrow A f \geq 0)$ corresponds to the cone of positive finite measures on $[-1,1]$. To extend Proposition 2.4 to distributions, let $\mathcal{D}[-1,1]$ be the Fréchet space of infinitely differentiable real functions on $[-1,1]$, supplied with the projective topology, analogous to the test function space $\mathcal{D}$ on $S^{d-1}$. Let $\mathcal{D}^{\prime}[-1,1]$ be its dual. All (generalized) functions on $[-1,1]$ are endowed with a tilde to distinguish them clearly from objects on $S^{d-1}$.

Proposition 2.5. The mapping $\Theta: \tilde{F} \mapsto A_{\tilde{F}}$ with

$$
\left(A_{\tilde{F}} \varphi\right)(u):=\tilde{F}_{(\alpha)}\left(R_{\alpha} \varphi\right)(u), \quad \varphi \in \mathcal{D}, u \in S^{d-1},
$$

is an isomorphism from $\mathcal{D}^{\prime}[-1,1]$ onto $\operatorname{End}(\mathcal{D})$.

Proof. We have $A_{\tilde{F}} \in \operatorname{End}(\mathcal{D})$, where the continuity of $A_{\tilde{F}}$ follows from (2.14) and the fact that $\tilde{F} \in \mathcal{D}^{\prime}[-1,1]$ is of finite order. The linearity of $\Theta$ is trivial.

To prove that $\Theta$ is surjective, define for $A \in \operatorname{End}(\mathcal{D})$ the distribution $\tilde{F} \in$ $\mathcal{D}^{\prime}[-1,1]$ by

$$
\tilde{F}(\tilde{\varphi}):=(A \tilde{\varphi}(\langle p, \cdot\rangle))(p), \quad \tilde{\varphi} \in \mathcal{D}[-1,1] .
$$

We will show $\Theta(\tilde{F})=A$. Due to (2.9) we get

$$
\left(A_{\tilde{F}} \varphi\right)(p)=\left(\tilde{F}_{(\alpha)}\left(R_{\alpha} \varphi\right)\right)(p)=\left(A_{(u)}\left(R_{\langle p, u\rangle} \varphi(p)\right)\right)(p)=(A \bar{\varphi})(p) .
$$

Due to Lemma 2.1, there is a sequence of finite rotation means of $\varphi$ about $p$ that converges to $\bar{\varphi}$ in $\mathcal{D}$. As $\varphi \mapsto(A \varphi)(p)$ is continuous and invariant with respect to rotations in $S O_{p^{\perp}}$, this implies

$$
\left(A_{\tilde{F}} \varphi\right)(p)=(A \bar{\varphi})(p)=(A \varphi)(p) .
$$

Using the fact that $S O_{d}$ acts transitively on $S^{d-1}$, we conclude that

$$
(\Theta \tilde{F})(\varphi)=A_{\tilde{F}} \varphi=A \varphi
$$

for all $\varphi \in \mathcal{D}$, so $\Theta(\tilde{F})=A$, as required. The injectivity of $\Theta$ follows from (2.16) if we put $\varphi:=\tilde{\varphi}(\langle p, \cdot\rangle), u:=p$ and use $\left(R_{\alpha} \varphi\right)(p)=\tilde{\varphi}(\alpha)$, where $\tilde{\varphi} \in \mathcal{D}[-1,1]$ is arbitrary.

$\Theta^{-1}(A)$ will be called the mixing distribution of $A \in \operatorname{End}(\mathcal{D})$. To give some examples, the mixing distribution of $R_{\alpha} \in \operatorname{End}(\mathcal{D})$ is a measure, namely the probabililty measure $\delta_{\alpha}$ supported by $\{\alpha\} \subset[-1,1]$. It follows from [1, Théorème 4.3] that the mixing distribution of $\Delta_{d} \in \operatorname{End}(\mathcal{D})$ is $(1-d) \delta_{1}^{\prime}$. 
A remark on the proof of Proposition 2.5 is in place here: When constructing the inverse image of $A$ under $\Theta$, we did not need the continuity of $A$ but only the continuity of $\varphi \mapsto(A \varphi)(p)$ to show $\tilde{F} \in \mathcal{D}^{\prime}[-1,1]$ and $A=\Theta\left(A_{\tilde{F}}\right) \in \operatorname{End}(\mathcal{D})$. This observation and Proposition 2.4 give the first part of the following corollary. The second part $(k=2)$ involves some tedious calculations which can be found in [11, pp. 35-37].

Corollary 2.6. Fix $k \in \mathbb{N}_{0}$. Let $A$ be a linear mapping from $\mathcal{D}$ into the space of real-valued functions on $S^{d-1}$ that commutes with rotations. If the linear functional

$$
\varphi \mapsto(A \varphi)(p)
$$

is continuous on $\mathcal{D}$ in the $\|\cdot\|_{\mathcal{D}_{k}}$-norm, then $A \in \operatorname{End}(\mathcal{D})$. For $k=0$, we have $A \in \operatorname{End}(\mathcal{C})$ (where we again write $A$ for the unique continuous extension of $A$ to $\mathcal{C})$.

If $k=2$, then

$$
\Theta^{-1}(A) \in \mathcal{E}_{2}^{\prime}[-1,1]
$$

where

$$
\begin{aligned}
\mathcal{E}_{2}^{\prime}[-1,1]:= & \left\{\tilde{\mu}_{0}+\frac{d}{d \alpha} \tilde{\mu}_{1}+\frac{d^{2}}{d \alpha^{2}} \tilde{\mu}_{2} \mid \tilde{\mu}_{0}, \tilde{\mu}_{1}, \tilde{\mu}_{2} \in \mathcal{M}[-1,1]\right. \text { and } \\
& \left.\int_{-1}^{1}\left(1-\alpha^{2}\right)^{-\rho}\left|\tilde{\mu}_{2}\right|(\alpha)<\infty \text { for all } 0 \leq \rho<1\right\}
\end{aligned}
$$

is a subspace of $\mathcal{D}_{2}^{\prime}[-1,1]$ (with $|\tilde{\mu}|$ denoting the variation measure of $\tilde{\mu}$ ).

Proposition 2.5 implies that $A \in \operatorname{End}(\mathcal{D})$ is self-adjoint,

$$
\int_{S^{d-1}}(A \varphi)(u) \psi(u) d \omega_{d-1}(u)=\int_{S^{d-1}} \varphi(u)(A \psi)(u) d \omega_{d-1}(u), \quad \varphi, \psi \in \mathcal{D},
$$

due to (2.10). As $\mathcal{D}$ is a dense subspace of $\mathcal{D}^{\prime}$, this implies that $A$ can be extended in a unique way to a continuous mapping from $\mathcal{D}^{\prime}$ into itself. This is due to the following standard argument, already used in the proof of Proposition 2.5. If $\mathcal{D} \subset$ $\mathcal{G} \subset \mathcal{D}^{\prime}$ and $A \in \operatorname{End}(\mathcal{G})$ satisfies (2.18), then the transpose map

$$
A^{\top}: \mathcal{G}^{\prime} \rightarrow \mathcal{G}^{\prime}, \quad F \mapsto F \circ A
$$

satisfies $A^{\top} \in \operatorname{End}\left(\mathcal{G}^{\prime}\right)$ and coincides with $A$ on $\mathcal{D}$ due to (2.18).

The extension of $A \in \operatorname{End}(\mathcal{D})$ to a mapping on $\mathcal{D}^{\prime}$ will again be denoted by $A$. We have $A \in \operatorname{End}\left(\mathcal{D}^{\prime}\right)$. Summarizing, we may write

$$
\operatorname{End}(\mathcal{D})=\operatorname{End}\left(\mathcal{D}^{\prime}\right) \quad \text { and } \quad \operatorname{End}(\mathcal{C})=\operatorname{End}(\mathcal{M}),
$$

in the sense that a mapping on the larger space becomes a mapping on the smaller space by restriction, and this operation is bijective. These statements are true in particular for $R_{\alpha} \in \operatorname{End}(\mathcal{C})$. Its extension to measures, given by

$$
\int_{S^{d-1}} f(u) d\left(R_{\alpha} \mu\right)(u)=\int_{S^{d-1}}\left(R_{\alpha} f\right)(u) d \mu(u), \quad f \in \mathcal{C}, \mu \in \mathcal{M},
$$

is an element of $\operatorname{End}(\mathcal{M})$.

Consider the case where the mixing distribution $\tilde{F}$ of $A \in \operatorname{End}(\mathcal{C})$ is not only a measure but a regular distribution. Then there is a function $\tilde{f}$ on $[-1,1]$, integrable 
with respect to the measure $\tilde{\mu}_{d}$ (given by (2.3) ), such that

$$
\tilde{F}(\tilde{\varphi})=\int_{-1}^{1} \tilde{\varphi}(\alpha) \tilde{f}(\alpha) d \tilde{\mu}_{d}(\alpha), \quad \tilde{\varphi} \in \mathcal{D}[-1,1] .
$$

As $A=A_{\tilde{\mu}},(2.15)$ then reads

$$
A f=\int_{S^{d-1}} f(u) \tilde{f}(\langle u, \cdot\rangle) d \omega_{d-1}(u), \quad f \in \mathcal{C} .
$$

It should be emphasized here that $\tilde{F}$ is not identified with a density function with respect to ordinary Lebesgue-measure on $[-1,1]$ but with respect to the measure $\tilde{\mu}_{d}$. This non-canonical identification of distributions is convenient here, as the weight function $\varpi_{d-1}\left(1-\alpha^{2}\right)^{(d-3) / 2}$ comes in naturally when introducing cylindrical coordinates on the sphere: For any $u \in S^{d-1}, \omega_{d-1}$ is the image measure of

$$
\tilde{\mu}_{d} \otimes \omega_{d-2}
$$

under the mapping $(\alpha, v) \mapsto \alpha u+\sqrt{1-\alpha^{2}} v$ on $[-1,1] \times S^{d-2}\left(u^{\perp}\right)$. Use of cylindrical coordinates on the sphere also yields the following: Let $A \in \operatorname{End}(\mathcal{M})$ with mixing measure $\tilde{\mu}$ be given. Then $\tilde{\mu}$ is a linear measure (i.e. a multiple of $\tilde{\mu}_{d}$ ) if and only if there is an $a \in \mathbb{R}$ such that

$$
A \mu=a\langle c(\mu), \cdot\rangle, \quad \mu \in \mathcal{M},
$$

where this equality is understood in the sense of distributions.

Fix $F \in \mathcal{D}^{\prime}, A \in \operatorname{End}\left(\mathcal{D}^{\prime}\right)$ and let $\tilde{F}=\Theta^{-1}(A)$. As the Fourier series $F \sim$ $\sum_{n=0}^{\infty} F_{n}$ converges in $\mathcal{D}^{\prime}$ to $F$, we have $A(F)=\sum_{n=0}^{\infty} A\left(F_{n}\right)$. (2.16) and (2.11) imply

$$
A(F)=\sum_{n=0}^{\infty} \lambda_{n}[A] \cdot F_{n}
$$

with the real numbers

$$
\lambda_{n}[A]=\tilde{F}_{(\alpha)}\left(P_{n}^{d}(\alpha)\right), \quad n \in \mathbb{N}_{0} .
$$

Mappings satisfying (2.22) are called multiplier transformations with multipliers $\lambda_{0}[A], \lambda_{1}[A], \lambda_{2}[A], \ldots$ That any $A \in \operatorname{End}(\mathcal{C})$ is a multiplier transformation has already been observed by Dunkl 22. $A$ is injective on $\mathcal{D}^{\prime}$ (or, equivalently, on $\mathcal{D}$ ) if and only if all its multipliers are non-zero. Due to (2.22) any two operators in $\operatorname{End}(\mathcal{D})$ commute.

2.3. Endomorphisms of convex bodies. Here we collect properties of endomorphisms of convex bodies that are well known or follow directly from the definitions. Some important results from convex geometry are also mentioned; as a general reference on this matter, we recommend SchNEIDER's book 18 .

Let $h(K, \cdot)=\max \{\langle x, \cdot\rangle \mid x \in K\}$ be the support function of $K \in \mathcal{K}^{d}$. Consider its Fourier expansion $h(K, \cdot) \sim \sum_{n=0}^{\infty} h_{n}(K, \cdot)$. Then $2 h_{0}(K, \cdot)$ is a constant function equal to the mean width $\bar{w}(K)$ of $K$. Furthermore, $h_{1}(K, \cdot)=\langle s(K), \cdot\rangle$, where

$$
s(K):=d \varpi_{d}^{-1} \int_{S^{d-1}} u h(K, u) d \omega_{d-1}(u)
$$

is the Steiner point of $K$. The convex body $K$ is a ball if and only if $h(K, \cdot)=$ $h_{0}(K, \cdot)+h_{1}(K, \cdot)$. It is a singleton if and only if $h(K, \cdot)=h_{1}(K, \cdot)$. 
If $S(K, \cdot) \sim \sum_{n=0}^{\infty} s_{n}(K, \cdot)$ is the Fourier expansion of the surface area measure of $K, \varpi_{d} s_{0}(K, \cdot)$ is the surface area of $K$ and

$$
s_{1}(K, \cdot)=o,
$$

as the centroid of any surface area measure coincides with $o$.

The basis for an analytical treatment of Minkowski-endomorphisms of convex bodies is the fact that the mapping $K \mapsto h(K, \cdot)$ is an isometric isomorphism from $\mathcal{K}^{d}$ into the subcone of $\mathcal{C}$ of all functions whose positive homogeneous extension (of degree one) is convex. A statement of similar importance for Blaschke-endomorphisms is based on Minkowski's existence theorem (see [18, Theorem 7.1.2]): Let $\mathcal{S}$ be the subcone of all positive measures in $\mathcal{M}$ having centroid $o$ and not being supported by any great subsphere. Minkowski's existence theorem states that any positive measure in $\mathcal{S}$ is the surface area measure of a uniquely determined convex body $K \in \mathcal{K}_{0}^{d}$. It can be shown that the mapping $K \mapsto S(K, \cdot)$ is a bicontinuous isomorphism from $\mathcal{K}_{0}^{d}$ into $\mathcal{S}$. In particular, integrals like the right-hand side of (1.7) are surface area measures of some convex body if $\tilde{\mu}$ is positive, and $R_{\alpha} S(K, \cdot)$ is a surface area measure for all $\alpha \in[-1,1]$.

We will also need the following property of the mixed volume in (1.8): If $K$ is a convex body with interior points, then $V(K, M[d-1])$ determines $K$ up to translation, if known for all $M \in \mathcal{K}_{0}^{d}$. Conversely, $M \in \mathcal{K}_{0}^{d}$ is uniquely determined by $K \mapsto V(K, M[d-1])$ on $\mathcal{K}_{0}^{d}$.

We turn to basic properties of Minkowski-endomorphisms. For $\Phi \in \operatorname{End}_{\mathrm{M}}\left(\mathcal{K}^{d}\right)$ we have

$$
\{o\}=\Phi(\{o\})=\Phi(\{x\}+\{-x\})=\Phi(\{x\})+\Phi(\{-x\}), \quad x \in \mathbb{R}^{d} .
$$

So, singletons are mapped to singletons. It is not difficult to see that $\Phi$ is homogeneous of degree one (see [15]) and maps balls to balls. The map $K \mapsto s(K)$ is continuous, motion-equivariant and Minkowski-additive (the Steiner point map is in fact characterized by these properties among all maps from $\mathcal{K}^{d}$ to $\mathbb{R}^{d}$ ). Hence, for any $a \in \mathbb{R}$, the map $K \mapsto\{a \cdot s(K)\}$ is a Minkowski-endomorphism. For $d \geq 3$, these are the only Minkowski-endomorphisms whose range consists only of singletons. This follows from a result in Section 3.1. For any $\Phi \in \operatorname{End}_{\mathrm{M}}\left(\mathcal{K}^{d}\right), d \geq 3$, there is a constant $\lambda_{1}[\Phi] \in \mathbb{R}$ with

$$
s(\Phi K)=\lambda_{1}[\Phi] \cdot s(K)
$$

for all $K \in \mathcal{K}^{d}$. (2.24), which can also be shown using elementary arguments (see [11]), has another interesting consequence: If $\Phi \in \operatorname{End}_{M}\left(\mathcal{K}^{d}\right)$ is given,

$$
\Phi^{\prime} K:=\Phi K+\left(1-\lambda_{1}[\Phi]\right) s(K), \quad K \in \mathcal{K}^{d},
$$

defines a $T_{d}$-equivariant Minkowski-endomorphism, i.e. an endomorphism in the sense of SCHNEIDER [15. Note that $\Phi^{\prime} K$ and $\Phi K$ coincide up to translation.

For $d=2$ we have the following representation theorem for Minkowski-endomorphisms, where $\vartheta_{\alpha} \in S O_{2}$ denotes the rotation at $o$ through angle $\alpha \in \mathbb{R}$ and positive orientation.

Proposition 2.7 (SCHNEIDER [16]). For any $\Phi \in \operatorname{End}_{\mathrm{M}}\left(\mathcal{K}^{2}\right)$ there is a finite signed Borel measure $\tilde{\mu}$ on $[-\pi, \pi)$ such that

$$
h(\Phi K, \cdot)=\int_{-\pi}^{\pi} h\left(\vartheta_{\alpha} K, \cdot\right) d \tilde{\mu}(\alpha), \quad K \in \mathcal{K}^{2} .
$$


$\tilde{\mu}$ is uniquely determined by (2.25). There are constants $c_{1}, c_{2} \in \mathbb{R}$, such that the measure

$$
\tilde{\mu}+\int_{(\cdot)}\left(c_{1} \cos \beta+c_{2} \sin \beta\right) d \beta
$$

is positive on $[-\pi, \pi)$.

Conversely, any signed measure $\tilde{\mu}$ with the property that (2.26) is positive for some $c_{1}, c_{2} \in \mathbb{R}$, defines a Minkowski-endomorphism by (2.25).

The Minkowski-endomorphism $\Phi$ in the previous proposition is translation invariant if and only if $\tilde{\mu}$ satisfies

$$
\int_{-\pi}^{\pi} \sin \alpha d \tilde{\mu}(\alpha)=\int_{-\pi}^{\pi} \cos \alpha d \tilde{\mu}(\alpha)=0
$$

In view of (1.6), we obtain the following.

Corollary 2.8. For any $\Psi \in \operatorname{End}_{\mathrm{B}}\left(\mathcal{K}_{0}^{2}\right)$ there is a finite positive Borel measure $\tilde{\mu} \neq 0$ on $[-\pi, \pi)$ such that

$$
S(\Psi K, \cdot)=\int_{-\pi}^{\pi} S\left(\vartheta_{\alpha} K, \cdot\right) d \tilde{\mu}(\alpha), \quad K \in \mathcal{K}_{0}^{2} .
$$

$\tilde{\mu}$ is uniquely determined up to addition of a measure of the form

$$
\int_{(\cdot)}\left(c_{1} \cos \beta+c_{2} \sin \beta\right) d \beta .
$$

Conversely, if $\tilde{\mu} \neq 0$ is a finite positive Borel measure on $[-\pi, \pi)$, then (2.27) defines a Blaschke-endomorphism $\Psi$.

Proof. Fix $\Psi \in \operatorname{End}_{\mathrm{B}}\left(\mathcal{K}_{0}^{2}\right)$. For $K \in \mathcal{K}^{2}$ we define

$$
\Phi K:=\lim _{n \rightarrow \infty} \Psi\left(K-s(K)+\frac{1}{n} B^{2}\right) .
$$

The limit here exists, as $\left(\Psi\left(K-s(K)+\frac{1}{n} B^{2}\right)\right)_{n \in \mathbb{N}}$ is a Cauchy sequence in the complete metric space $\mathcal{K}^{2}$. Due to (1.6), this gives rise to a $T_{2}$-invariant Minkowskiendomorphism $\Phi$. Conversely, any $T_{2}$-invariant Minkowski-endomorphism induces a Blaschke-endomorphism if restricted to $\mathcal{K}_{0}^{2}$.

The result now follows easily from Proposition 2.7 if we note that $c(S(K, \cdot))=o$ can be rewritten as

$$
\int_{-\pi}^{\pi} S\left(\vartheta_{\alpha} K, \cdot\right) \cos \alpha d \alpha=\int_{-\pi}^{\pi} S\left(\vartheta_{\alpha} K, \cdot\right) \sin \alpha d \alpha=0 .
$$

Returning to arbitrary dimension, we note that $\Psi \in \operatorname{End}_{\mathrm{B}}\left(\mathcal{K}_{0}^{d}\right)$ is positive homogeneous of degree one and maps balls in $\mathcal{K}_{0}^{d}$ to concentric balls.

\section{Proofs of the Main theorems}

3.1. Minkowski-endomorphisms. We are now proving the main results, starting with Theorem 1.3 .

Proof of Theorem 1.3. Assume $d \geq 3$ and fix $\Phi \in \operatorname{End}_{\mathrm{M}}\left(\mathcal{K}^{d}\right)$. Let

$$
\mathcal{G}:=\left\{h(K, \cdot)-h(M, \cdot) \mid K, M \in \mathcal{K}^{d}\right\} \subset \mathcal{C}
$$


be the vector space of differences of support functions. We interpret $\Phi$ as a rotationcommuting mapping from $\mathcal{G}$ to $\mathcal{C}$ by setting

$$
\Phi(h(K, \cdot)-h(M, \cdot)):=h(\Phi K, \cdot)-h(\Phi M, \cdot), \quad K, M \in \mathcal{K}^{d},
$$

which is well defined due to the Minkowski-additivity of $\Phi$. Any function $\varphi \in \mathcal{D}_{2}$ can be written as the difference

$$
\varphi=h\left(M_{\varphi}, \cdot\right)-h\left(\alpha_{\varphi} B^{d}, \cdot\right)
$$

of two support functions, where $\alpha_{\varphi} \geq 0$ and (consequently) the body $M_{\varphi}$ depend continuously on $\varphi$ in the $\mathcal{D}_{2}$-norm; see e.g. [18, Lemma 1.7.9]. Hence $\mathcal{D}_{2} \subset \mathcal{G}$ and the restriction $A:=\left.\Phi\right|_{\mathcal{D}_{2}}$ is a continuous mapping from $\mathcal{D}_{2}$ into $\mathcal{C}$. Corollary 2.6 implies $A \in \operatorname{End}(\mathcal{D})$ and $\tilde{F}:=\Theta^{-1}(A) \in \mathcal{E}_{2}^{\prime}[-1,1]$. For all $K \in \mathcal{K}^{d}$ with $h(K, \cdot) \in \mathcal{D}$, we have due to Proposition 2.5

$$
h(\Phi K, \cdot)=A h(K, \cdot)=\tilde{F}_{(\alpha)}\left(R_{\alpha} h(K, \cdot)\right) .
$$

(3.2) is also true for arbitrary $K \in \mathcal{K}^{d}$, if $A$ and $R_{\alpha}$ are considered as mappings on $\mathcal{D}^{\prime}$. This follows from the fact that the set of support functions in $\mathcal{D}$ is dense in the cone of arbitrary support functions, supplied with the maximum-norm. Hence we have shown the representation (1.2) in Theorem 1.3. (3.1) and the first equality in (3.2) show that $\Phi$ determines $A$ on $\mathcal{D}$. Hence $\tilde{F}=\Theta^{-1}(A)$ is uniquely determined by $\Phi$.

The equivalence i) is a simple consequence of this uniqueness and

$$
R_{\alpha} h(-K, \cdot)=R_{-\alpha} h(K, \cdot) .
$$

If $\Phi$ is uniformly continuous, the above constructed map $A: \mathcal{D}_{2} \rightarrow \mathcal{C}$ is continuous, even if both spaces are endowed with the $\|\cdot\|_{\infty}$-norm. Corollary 2.6 now implies $A \in \operatorname{End}(\mathcal{C})$, and Proposition 2.4 shows that $\tilde{F}=\Theta^{-1}(A) \in \mathcal{M}[-1,1]$. As the converse implication is clear, this gives equivalence ii) in Theorem 1.3 .

Finally, if $\Phi$ is monotonic, it is also uniformly continuous and $A \in \operatorname{End}(\mathcal{C})$ is monotonic, too. The remark after Proposition 2.4 now implies the non-trivial implication of the equivalence iii).

If $\alpha \mapsto\left(R_{\alpha} h(K, \cdot)\right)(u), K \in \mathcal{K}^{d}, u \in S^{d-1}$, is not sufficiently smooth, (1.2) must be interpreted in the sense of distributions:

$$
\int_{S^{d-1}} h(\Phi K, u) \varphi(u) d \omega_{d-1}(u)=\int_{S^{d-1}} h(K, u) \tilde{F}_{(\alpha)}\left(R_{\alpha} \varphi\right)(u) d \omega_{d-1}(u)
$$

for all test functions $\varphi \in \mathcal{D}$.

It follows from (1.2) that $\Phi$ is a multiplier transformation: For $K \in \mathcal{K}^{d}$ with $h(K, \cdot) \sim \sum_{n=0}^{\infty} h_{n}(K, \cdot)$ being the Fourier expansion of its support function, we have

$$
h(\Phi K, \cdot) \sim \sum_{n=0}^{\infty} \lambda_{n}[\Phi] h_{n}(K, \cdot) .
$$

Here, the multipliers

$$
\lambda_{n}[\Phi]:=\tilde{F}_{(\alpha)}\left(P_{n}^{d}(\alpha)\right)
$$

can be expressed in terms of the mixing distribution $\tilde{F}$ of $\Phi$. The first two multipliers have a simple geometric interpretation:

$$
\bar{w}(\Phi K)=\lambda_{0}[\Phi] \cdot \bar{w}(K), \quad K \in \mathcal{K}^{d},
$$


in particular, $\Phi B^{d}=\lambda_{0}[\Phi] \cdot B^{d}$, and

$$
s(\Phi K)=\lambda_{1}[\Phi] \cdot s(K), \quad K \in \mathcal{K}^{d},
$$

which is (2.24).

SCHNEIDER [15] has shown the multiplier property of $\Phi$ with different arguments. He has additionally proven

$$
\left|\lambda_{n}[\Phi]\right| \leq \lambda_{0}[\Phi], \quad n=2,3, \ldots
$$

From (3.4) he concluded that all Minkowski-endomorphisms that commute with affine maps, or are surjective, or preserve the volume, are trivial. It is also well known that injectivity of $\Phi$ can be deduced directly from its multipliers: We note the following corollary for later reference.

Corollary 3.1. $\Phi \in \operatorname{End}_{\mathrm{M}}\left(\mathcal{K}^{d}\right)$ is injective if and only if all its multipliers are non-zero.

If $\Phi \in \operatorname{End}_{\mathrm{M}}\left(\mathcal{K}^{d}\right)$ is such that its mixing distribution is regular and can be identified with the function $\tilde{f}(\alpha)=d \varpi_{d}^{-1} \alpha$, (3.2), (2.21) and (2.23) imply

$$
\Phi K=\{s(K)\}, \quad K \in \mathcal{K}^{d} .
$$

Hence, the linear measures defined before Theorem 1.4 are precisely the mixing measures of the Minkowski-endomorphisms $K \mapsto\{a \cdot s(K)\}, a \in \mathbb{R}$.

We turn to the proof of Theorem [1.4. It is split into two main propositions and an auxiliary lemma.

Proposition 3.2. Assume $K \in \mathcal{K}^{d}, d \geq 3$, and $\alpha \in[-1,1]$. Then $R_{\alpha} h(K, \cdot)$ is the support function of a convex body $K_{\alpha} . K \mapsto K_{\alpha}$ is a Minkowski-endomorphism.

Proof. Let $f$ be the positive homogeneous extension (of degree one) of $h(K, \cdot)$. To show that $R_{\alpha} f$ is a support function, we have to prove that its positive homogeneous extension

$$
\left(R_{\alpha} f\right)(x):=\varpi_{d-1}^{-1} \int_{S\left(x^{\perp}\right)} f\left(\alpha x+\sqrt{1-\alpha^{2}}\|x\| v\right) d \omega_{d-2}(v), \quad x \neq 0,
$$

(and $\left.\left(R_{\alpha} f\right)(0):=0\right)$ is convex. It is enough to show convexity of the restriction of $R_{\alpha} f$ to an arbitrary two-dimensional plane $L \subset \mathbb{R}^{d}$. Let $\vartheta_{L} \in S O_{d}$ be the rotation that fixes $L^{\perp}$ pointwise and rotates any vector in $L$ by the angle $\pi / 2$ (with arbitrary but fixed orientation).

For all $z \in \mathbb{R}^{d}, \tau, \sigma \in \mathbb{R}$, the function

$$
\begin{aligned}
g(x) & =g_{z, \tau, \sigma}(x) \\
& :=f\left(\tau x+\sigma \vartheta_{L} x+\|x\| z\right)+f\left(\tau x+\sigma \vartheta_{L} x-\|x\| z\right), \quad x \in L,
\end{aligned}
$$

is convex on $L$. The special case $\tau=1, \sigma=0$ of this statement was shown in Schneider [15, Proposition 2.5]. The proof of the general case is the same; in particular the weights $\alpha, \beta, \gamma$ in the proof of Schneider's Proposition 2.5 remain unchanged. 
Now consider the case $d=3$. Let $L=v^{\perp}$ for some $v \in S^{2}$. The convexity of $R_{\alpha} f$ on $L$ now follows if we introduce cylindrical coordinates on $S\left(x^{\perp}\right), x \in L \backslash\{o\}$ :

$$
\begin{aligned}
2 \pi\left(R_{\alpha} f\right)(x) & =\int_{S\left(x^{\perp}\right)} f\left(\alpha x+\sqrt{1-\alpha^{2}}\|x\| u\right) d \omega_{1}(u) \\
& =\int_{-1}^{1} g_{z, \tau, \sigma}(x) \frac{d \beta}{\sqrt{1-\beta^{2}}}
\end{aligned}
$$

with $z=\sqrt{1-\alpha^{2}} \sqrt{1-\beta^{2}} v, \tau=\alpha, \sigma=\sqrt{1-\alpha^{2}} \beta$.

It remains to consider the case $d>3$. Introducing cylindrical coordinates, we see that

$$
\begin{aligned}
& \varpi_{d-1}\left(R_{\alpha} f\right)(x)=\int_{S\left(x^{\perp}\right)} f\left(\alpha x+\sqrt{1-\alpha^{2}}\|x\| v\right) d \omega_{d-2}(v) \\
&=\int_{-1}^{1} \int_{S\left(L^{\perp}\right)} f\left(\alpha x+\sqrt{1-\alpha^{2}} \beta \vartheta_{L} x+\sqrt{1-\alpha^{2}} \sqrt{1-\beta^{2}}\|x\| v\right) \\
& d \omega_{d-3}(v)\left(1-\beta^{2}\right)^{(d-5) / 2} d \beta
\end{aligned}
$$

holds for $x \in L$. As $\omega_{d-3}$ is invariant under reflection $v \mapsto-v$, the convexity of this function (on $L$ ) follows from the convexity of $g$.

That $K \mapsto K_{\alpha}$ is a Minkowski-endomorphism now follows easily.

We have defined the centroid of a measure $\mu \in \mathcal{M}$ in (2.8). Identifying $f \in \mathcal{C}$ with the measure having $f$ as (signed) density with respect to spherical Lebesguemeasure, we write $c(f)=\int_{S^{d-1}} u f(u) d \omega_{d-1}(u)$. The definition is such that $d \varpi_{d}^{-1} c(h(K, \cdot))$ is just the Steiner point of $K \in \mathcal{K}^{d}$.

Proposition 3.3. Let $\tilde{\mu}$ be the mixing measure of $A \in \operatorname{End}(\mathcal{C})$. If for all $f \in \mathcal{C}$, we have

$$
f \geq 0, c(f)=o \quad \Rightarrow \quad A f \geq 0,
$$

then $\tilde{\mu}$ is positive up to addition of a linear measure.

Proof. We adapt an argument used in SCHneIDER [16] when characterizing Minkowski-endomorphisms on $\mathcal{K}^{2}$. Fix $A \in \operatorname{End}(\mathcal{C})$ with mixing measure $\tilde{\mu}$. We have to show that there is a constant $c \in \mathbb{R}$ such that

$$
\tilde{\mu}+c \int_{(\cdot)} \alpha d \tilde{\mu}_{d}(\alpha)
$$

is a positive measure.

At first, we consider the case where

$$
\frac{d \tilde{\mu}}{d \tilde{\mu}_{d}}=\tilde{\varphi} \in \mathcal{D}[-1,1]
$$

Then, the measure in (3.7) is positive if and only if

$$
\tilde{\varphi}(\alpha)+c \cdot \alpha \geq 0
$$

for all $\alpha \in[-1,1]$. We have $\varphi(u):=\tilde{\varphi}(\langle p, u\rangle) \in \mathcal{D}$. The change from integration with respect to cylindrical coordinates to ordinary integration on the sphere gives

$$
(A f)(p)=\int_{-1}^{1} \tilde{\varphi}(\alpha)\left(R_{\alpha} f\right)(p) d \tilde{\mu}_{d}(\alpha)=\int_{S^{d-1}} \varphi(u) f(u) d \omega_{d-1}(u) .
$$


The assumption (3.6) implies

$$
\int_{S^{d-1}} \varphi(u) d \mu(u) \geq 0
$$

for all positive $\mu \in \mathcal{M}$ with $c(\mu)=o$, as any measure can (weakly) be approximated by continuous functions. Due to (3.1), there is an $\alpha=\alpha_{\varphi}>0$ such that $\varphi+\alpha$ is the support function of a convex body $M=M_{\varphi}$. It may be assumed that $M$ has interior points. Let $\rho>0$ be the maximal radius of a ball contained in $M$ ( $\rho$ is the so-called inradius of $M)$ and let $x \in \mathbb{R}^{d}$ be such that $B^{d} \subset(1 / \rho)(M+x)$. As $M$ is a body of revolution with axis parallel to $p$, we have $x=c \cdot p$ for some $c \in \mathbb{R}$. The convex hull of

$$
T:=\left(\operatorname{bd} B^{d}\right) \cap(1 / \rho) \operatorname{bd}(M+c p) \subset S^{d-1}
$$

contains $o$ in its relative interior (this follows from the maximality of $\rho$ ). Hence, there exists a positive measure $\mu_{0} \in \mathcal{M}, \mu_{0} \neq 0$, with support in $T$ and $c\left(\mu_{0}\right)=o$. (3.9) implies

$$
\begin{aligned}
\mu_{0}\left(S^{d-1}\right) & =\int_{T} h\left(B^{d}, u\right) d \mu_{0}(u)=\frac{1}{\rho} \int_{T}(h(M, u)+c\langle p, u\rangle) d \mu_{0}(u) \\
& =\frac{1}{\rho} \int_{S^{d-1}} h(M, u) d \mu_{0}(u)=\frac{1}{\rho} \int_{S^{d-1}}(\varphi(u)+\alpha) d \mu_{0}(u) \geq \frac{\alpha}{\rho} \mu_{0}\left(S^{d-1}\right) .
\end{aligned}
$$

We conclude $1 \geq \frac{\alpha}{\rho}$ and

$$
\frac{1}{\rho}(\varphi(u)+\alpha+c\langle p, u\rangle)=h\left(\frac{1}{\rho}(M+c p), u\right) \geq 1 \geq \frac{\alpha}{\rho}, \quad u \in S^{d-1} .
$$

This implies $\varphi(u)+c\langle p, u\rangle \geq 0$, for all $u \in S^{d-1}$, and (3.8) holds.

Now we allow the mixing measure $\tilde{\mu} \in \mathcal{M}[-1,1]$ of $A$ to be arbitrary. Consider the smoothened operators $A_{n}:=B_{n} \circ A$, where the mixing measure of $B_{n} \in \operatorname{End}(\mathcal{C})$ has the $\tilde{\mu}_{d}$-density $\tilde{\psi}_{n} \in \mathcal{D}[-1,1], \tilde{\psi}_{n} \geq 0, n=0,1,2, \ldots A_{n}$ satisfies (3.6). The additional assumption that $\left(\tilde{\psi}_{n}\right)$ is chosen in such a way that

$$
\lim _{n \rightarrow \infty} \int_{(\cdot)} \tilde{\psi}_{n}(\alpha) d \tilde{\mu}_{d}(\alpha)=\delta_{1}
$$

(in the sense of weak convergence in $\mathcal{M}[-1,1]$ ) implies

$$
\lim _{n \rightarrow \infty} A_{n} f=A f
$$

for all $f \in \mathcal{C}$. According to Lemma 3.4 below, the mixing measure of $A_{n}$ has a $\tilde{\mu}_{d}$-density $\tilde{\varphi}_{n} \in \mathcal{D}[-1,1]$. We can apply the first part of this proof to $A_{n}$ and conclude that there are constants $c_{n}$ such that

$$
\tilde{\varphi}_{n}(\alpha)+c_{n} \cdot \alpha \geq 0, \quad \alpha \in[-1,1]
$$

for all $n=0,1,2, \ldots$. The sequence $\left(c_{n}\right)$ is bounded: Let $f \in \mathcal{C}$ be non-negative and $u \in S^{d-1}$ such that $\langle u, c(f)\rangle>0$. It follows from (2.21) and (3.11) that

$$
\begin{aligned}
\left(A_{n} f\right)(u) & =\int_{S^{d-1}} f(v) \tilde{\varphi}_{n}(\langle u, v\rangle) d \omega_{d-1}(v) \\
& \geq-c_{n} \int_{-1}^{1} f(v)\langle u, v\rangle d \omega_{d-1}(v) \\
& =-c_{n} \frac{\varpi_{d}}{d}\langle u, c(f)\rangle .
\end{aligned}
$$


In view of (3.10), this shows that the sequence $\left(c_{n}\right)$ is bounded from below. If $u$ is replaced by $-u$, the same arguments show that $\left(c_{n}\right)$ is bounded from above, too. Without loss of generality, we assume $c_{n} \rightarrow c$ as $n \rightarrow \infty$. We may in addition assume that the sequence of the positive measures

$$
\int_{(\cdot)}\left(\tilde{\varphi}_{n}(\alpha)+c_{n} \alpha\right) d \tilde{\mu}_{d}(\alpha)
$$

converges to a positive measure $\tilde{\nu} \in \mathcal{M}[-1,1]$, as the total masses

$$
\begin{aligned}
\int_{-1}^{1}\left(\tilde{\varphi}_{n}(\alpha)+c_{n} \alpha\right) d \tilde{\mu}_{d}(\alpha) & =\int_{-1}^{1} \tilde{\varphi}_{n}(\alpha) d \tilde{\mu}_{d}(\alpha) \\
& =\int_{-1}^{1}\left(R_{\alpha} 1\right)(p) \tilde{\varphi}_{n}(\alpha) d \tilde{\mu}_{d}(\alpha)=\left(A_{n} 1\right)(p)
\end{aligned}
$$

are bounded by a constant due to (3.10). Using (3.10) and the weak convergence of the measures involved, we conclude

$$
\tilde{\mu}+c \int_{(\cdot)} \alpha d \tilde{\mu}_{d}(\alpha)=\tilde{\nu}
$$

which yields the assertion.

For the following lemma, used in the previous proof, we call a function $\tilde{f}$ a mixing function of $A \in \operatorname{End}(\mathcal{C})$ if the mixing measure $\tilde{\mu}$ of $A$ satisfies $d \tilde{\mu}=\tilde{f} d \tilde{\mu}_{d}$.

Lemma 3.4. Let $A \in \operatorname{End}(\mathcal{C})$ be given. If there exists a mixing function of $B \in$ $\operatorname{End}(\mathcal{C})$ in $\mathcal{D}[-1,1]$, then there is a mixing function of $B \circ A$ in $\mathcal{D}[-1,1]$.

Proof. Let $\tilde{\nu}$ be the mixing measure of $B \circ A \in \operatorname{End}(\mathcal{C})$ and let $\tilde{\varphi} \in \mathcal{D}[-1,1]$ be a mixing function of $B$. For arbitrary $\tilde{f} \in \mathcal{C}[-1,1]$ the definition of a mixing measure implies

$$
((B \circ A) \tilde{f}(\langle p, \cdot\rangle))(p)=\int_{-1}^{1} \tilde{f}(\alpha) d \tilde{\nu}(\alpha) .
$$

The expression on the left-hand side can also be transformed using $B \circ A=A \circ B$ and (2.21):

$$
\begin{aligned}
((B \circ A) \tilde{f}(\langle p, \cdot\rangle))(p) & =A\left(\int_{S^{d-1}} \tilde{f}(\langle p, u\rangle) \tilde{\varphi}(\langle u, \cdot\rangle) d \omega_{d-1}(u)\right)(p) \\
& =\int_{S^{d-1}} \tilde{f}(\langle p, u\rangle) A(\tilde{\varphi}(\langle u, \cdot\rangle))(p) d \omega_{d-1}(u) .
\end{aligned}
$$

Put $g(u):=A(\tilde{\varphi}(\langle u, \cdot\rangle))(p)$. For every $u \in S^{d-1}$, there exists a rotation $\vartheta \in S O_{d}$ with $\vartheta u=p$ and $\vartheta p=u$, as $d \geq 3$. The operator $A$ commutes with rotations, so

$$
g(u)=A(\tilde{\varphi}(\langle u, \cdot\rangle))(\vartheta u)=A(\tilde{\varphi}(\langle u, \vartheta \cdot\rangle))(u)=A(\tilde{\varphi}(\langle p, \cdot\rangle))(u)
$$

is a zonal function. Moreover we have $g \in \mathcal{D}$, as the restriction of $A$ to $\mathcal{D}$ is in $\operatorname{End}(\mathcal{D})$ due to Propositions 2.4 and 2.5 . So, there is a $\tilde{\psi} \in \mathcal{D}[-1,1]$ with $g=\tilde{\psi}(\langle p, \cdot\rangle)$. Substitution of $g$ into (3.13) and comparison with (3.12) implies

$$
\int_{-1}^{1} \tilde{f}(\alpha) d \tilde{\nu}(\alpha)=\int_{-1}^{1} \tilde{f}(\alpha) \tilde{\psi}(\alpha) d \tilde{\mu}_{d}(\alpha), \quad \tilde{f} \in \mathcal{C}[-1,1] .
$$

Hence, $\tilde{\psi} \in \mathcal{D}[-1,1]$ is a mixing function of $B \circ A$. 
Proof of Theorem 1.4. Let $\Phi \in \operatorname{End}_{\mathrm{M}}\left(\mathcal{K}^{d}\right)$ be weakly monotonic. It follows from (2.24) and the Lipschitz-continuity of the Steiner point map that $\Phi$ is uniformly continuous. Let $\tilde{\mu} \in \mathcal{M}[-1,1]$ be the mixing measure of $\Phi$ which exists due to Theorem 1.3 i). The operator $A \in \operatorname{End}(\mathcal{C})$ with mixing measure $\tilde{\mu}$ (i.e. $A=\Theta(\tilde{\mu})$ ) satisfies the assumptions of Proposition 3.3, (3.1) and the weak monotony of $\Phi$ imply (3.6) for all $f \in \mathcal{D}_{2}$ and by approximation in the maximum-norm for all $f \in \mathcal{C}$. Proposition 3.3 now shows that $\tilde{\mu}$ is positive up to addition of a linear measure.

To show the converse let $\tilde{\mu}$ be a measure on $[-1,1]$ and $c \in \mathbb{R}$ a constant such that

$$
\tilde{\nu}:=\tilde{\mu}+c \int_{(\cdot)} \alpha d \tilde{\mu}_{d}(\alpha)
$$

is a positive measure. Proposition 3.2 implies that there is $\chi \in \operatorname{End}_{M}\left(\mathcal{K}^{d}\right)$ with mixing measure $\tilde{\nu} . \quad \chi$ is monotonic due to Theorem [1.3 ii). We have seen that $c \int_{(\cdot)} \alpha d \tilde{\mu}_{d}(\alpha)$ is the mixing measure of the Minkowski-endomorphism $K \mapsto$ $\{a \cdot s(K)\}$ for some suitable $a \in \mathbb{R}$. Summarizing, we have for arbitrary $K \in \mathcal{K}^{d}$

$$
\int_{-1}^{1} R_{\alpha} h(K, \cdot) d \tilde{\mu}(\alpha)=h(\chi K, \cdot)+h(\{-a \cdot s(K)\}, \cdot),
$$

which is obviously the support function of the convex body $K^{\prime}=\chi K-a \cdot s(K)$. The mapping $\Phi: K \mapsto K^{\prime}$ is a Minkowski-endomorphism, and it is weakly monotonic, as $\Phi K=\chi K$ if $s(K)=o$.

3.2. Blaschke-endomorphisms. First, a result analogous to Proposition 3.2 is required with the support function replaced by the surface area measure.

Lemma 3.5. Assume $K \in \mathcal{K}_{0}^{d}, d \geq 3$, and $\alpha \in[-1,1]$. Then $R_{\alpha} S(K, \cdot)$ is the surface area measure of a convex body $K_{\alpha} \in \mathcal{K}_{0}^{d} . K \mapsto K_{\alpha}$ is a Blaschkeendomorphism.

Proof. The measure $\mu:=R_{\alpha} S(K, \cdot)$ is positive. We may assume $\alpha \geq 0$, as $R_{-\alpha} S(K, \cdot)=R_{\alpha} S(-K, \cdot)$. According to Minkowski's existence theorem, we have to show that $c(\mu)=o$ and that $\mu$ is not supported by a great subsphere. For arbitrary $u \in S^{d-1},(2.20)$ implies

$$
\begin{aligned}
\langle c(\mu), u\rangle & =\int_{S^{d-1}} P_{1}^{d}(\langle v, u\rangle) d\left(R_{\alpha} S(K, \cdot)\right)(v) \\
& =\int_{S^{d-1}}\left(R_{\alpha} P_{1}^{d}(\langle u, \cdot\rangle)\right)(v) d S(K, v) .
\end{aligned}
$$

In view of (2.11), as $u$ was arbitrary, we conclude that

$$
c(\mu)=\alpha \cdot c(S(K, \cdot))=o .
$$

Let $u^{+}$denote the open half-space bounded by $u^{\perp}$ and containing the unit vector $u$. Then

$$
\mu\left(S^{d-1} \cap u^{+}\right)=\int_{S^{d-1}} \mathbf{1}_{u^{+}}(v) d\left(R_{\alpha} S(K, \cdot)\right)(v)=\int_{S^{d-1}}\left(R_{\alpha} \mathbf{1}_{u^{+}}\right)(v) d S(K, v) .
$$

For any $v \in u^{+}$, we have $\left(R_{\alpha} \mathbf{1}_{u^{+}}\right)(v) \geq 1 / 2$, so

$$
\mu\left(S^{d-1} \cap u^{+}\right) \geq \frac{1}{2} S\left(K, S^{d-1} \cap u^{+}\right)>0,
$$


implying that $\mu$ is not supported by a great subsphere and must be the surface area measure of a convex body $K_{\alpha} \in \mathcal{K}_{0}^{d}$. It is clear that $K \mapsto K_{\alpha}$ is a Blaschkeendomorphism.

Proof of Theorem 1.7. Let $\Psi \in \operatorname{End}_{\mathrm{B}}\left(\mathcal{K}_{0}^{d}\right), d \geq 3$, be given. For any $\mu \in \mathcal{M}$, there are convex bodies $K_{+}, K_{-} \in \mathcal{K}_{0}^{d}$ such that

$$
\mu-d \varpi_{d}^{-1} \int_{(\cdot)}\langle c(\mu), u\rangle d \omega_{d-1}(u)=S\left(K_{+}, \cdot\right)-S\left(K_{-}, \cdot\right),
$$

as the measure on the left has centroid $o$. The definition

$$
G(\mu):=S\left(\Psi K_{+}, \cdot\right)-S\left(\Psi K_{-}, \cdot\right)
$$

does not depend on the particular choice of $K_{+}$and $K_{-}$due to the Blaschke additivity of $\Psi$. This gives rise to a linear operator $G: \mathcal{M} \rightarrow \mathcal{M}$ that commutes with rotations. We have

$$
S(\Psi K, \cdot)=G(S(K, \cdot)), \quad K \in \mathcal{K}_{0}^{d} .
$$

Let $\delta_{u}$ be the probability measure on $S^{d-1}$ supported by $\{u\}$. The operator $A$ from $\mathcal{D}$ into the space of real functions on $S^{d-1}$, given by

$$
(A \varphi)(u):=\int_{S^{d-1}} \varphi(v) d\left(G \delta_{u}\right)(v), \quad \varphi \in \mathcal{D}, u \in S^{d-1},
$$

satisfies all assumptions of Corollary 2.6 (with $k=0$ ) and hence $A \in \operatorname{End}(\mathcal{C})$. The unique continuous extension of $A$ to $\mathcal{M}$ is in $\operatorname{End}(\mathcal{M})$ and satisfies

$$
\int_{S^{d-1}} \varphi(v) d\left(A \delta_{u}\right)(v)=\int_{S^{d-1}}(A \varphi)(v) d \delta_{u}(v)=(A \varphi)(u)=\int_{S^{d-1}} \varphi(v) d\left(G \delta_{u}\right)(v),
$$

so $A$ and $G$ coincide on the family of finitely supported signed measures. As the surface area measure of any convex polytope is finitely supported, we have for all polytopes $K \in \mathcal{K}_{0}^{d}$

$$
S(\Psi K, \cdot)=A(S(K, \cdot)),
$$

due to (3.15). (3.16) even holds for arbitrary $K \in \mathcal{K}_{0}^{d}$, as both sides depend weakly continuously on $K$. (3.16) now implies (1.7), where $\tilde{\mu} \in \mathcal{M}$ is the mixing measure of $A$. The fact that there exists a linear measure $\tilde{\nu}$ such that $\tilde{\mu}+\tilde{\nu}$ is positive follows from Proposition 3.3 , as any non-vanishing continuous function $f \geq 0$ with $c(f)=o$ can be interpreted as density of the surface area measure of some convex body. If $\tilde{\mu}+\tilde{\nu}$ was the zero-measure, $\tilde{\mu}$ would be a linear measure. Then

$$
\int_{-1}^{1} \alpha R_{\alpha} S(K, \cdot) d \tilde{\mu}_{d}(\alpha)=\langle c(S(K, \cdot)), \cdot\rangle=0, \quad K \in \mathcal{K}_{0}^{d},
$$

which holds in the sense of distributions, would show that $S(\Psi K, \cdot)=0$ for all $K \in \mathcal{K}_{0}^{d}$, contradicting $\Psi: \mathcal{K}_{0}^{d} \rightarrow \mathcal{K}_{0}^{d}$.

We now show that $\tilde{\mu}$ is determined up to addition of a linear measure by (1.7). Fix $\Psi \in \operatorname{End}_{\mathrm{B}}\left(\mathcal{K}_{0}^{d}\right)$. If $\tilde{\mu}$ satisfies (1.7) and $A \in \operatorname{End}(\mathcal{M})$ has mixing measure $\tilde{\mu}$, then (3.16) holds. Hence, $A f$ is determined by $\Psi$ for all non-vanishing functions $f \in \mathcal{C}$ with $f \geq 0$ and $c(f)=o$. The condition $f \geq 0$ can be omitted here, showing that all multipliers of $A$ with the exception of $\lambda_{1}[A]$ are determined by $\Psi$. This implies that $\tilde{\mu}$ is determined up to addition of a linear measure by (1.7).

Assume now that $\tilde{\mu}$ is a signed measure and $\tilde{\nu}$ is a linear measure such that $\tilde{\mu}+\tilde{\nu}$ is positive and non-zero. The fact that the right-hand side of (1.7) is the 
surface area measure of some convex body follows from Lemma 3.5 and (3.17). The remaining assertions are clear.

In contrast to mixing measures of weakly monotonic Minkowski-endomorphisms, mixing measures of Blaschke-endomorphisms are only determined up to addition of a linear measure. Similar to the case of Minkowski-endomorphisms, (1.2) implies that $\Psi \in \operatorname{End}_{\mathrm{B}}\left(\mathcal{K}_{0}^{d}\right)$ is a multiplier transformation: Let $\tilde{\mu}$ be a mixing measure of $\Psi$ and $K \in \mathcal{K}_{0}^{d}$. If $S(K, \cdot) \sim \sum_{n=0}^{\infty} s_{n}(K, \cdot)$ is the Fourier-expansion of its surface area measure, we have

$$
S(\Psi K, \cdot) \sim \sum_{n=0}^{\infty} \lambda_{n}[\Psi] s_{n}(K, \cdot)
$$

with

$$
\lambda_{n}[\Psi]:=\int_{-1}^{1} P_{n}^{d}(\alpha) d \tilde{\mu}(\alpha) .
$$

It is easily checked that

$$
S(\Psi K)=\lambda_{0}[\Psi] \cdot S(K), \quad K \in \mathcal{K}_{0}^{d},
$$

holds, where $S(K):=S\left(K, S^{d-1}\right)$ is the surface area of $K$. In particular, $\lambda_{0}[\Psi]$ is positive: It is the surface area of the ball $\omega_{d}^{-1 /(d-1)} \Psi B^{d}$. As $s_{1}(K, \cdot)=0$, the value of $\lambda_{1}[\Psi]$ does not contribute to the right-hand side of (3.18). This corresponds to the fact that the mixing measure of $\Psi$ is only determined up to addition of a linear measure.

Corollary 3.6. $\Psi \in \operatorname{End}_{\mathrm{B}}\left(\mathcal{K}_{0}^{d}\right)$ is injective if and only if $\lambda_{n}[\Psi] \neq 0$ holds for all $n \in \mathbb{N}_{0}, n \neq 1$.

3.3. Adjoint endomorphisms and applications. To prove Theorem 1.8, we note that $\Phi \in \operatorname{End}_{\mathrm{M}}\left(\mathcal{K}^{d}\right)$ is $T_{d}$-equivariant if and only if $\lambda_{1}[\Phi]=1$. This follows from

$$
h(\Phi\{x\}, \cdot)=h_{1}(\Phi\{x\}, \cdot)=\lambda_{1}[\Phi] h_{1}(\{x\}, \cdot)=\lambda_{1}[\Phi] h(\{x\}, \cdot)
$$

and the Minkowski-additivity of $\Phi . \Phi$ is non-degenerate if and only if $\lambda_{0}[\Phi]$ is positive.

If $\tilde{\mu}$ is the mixing measure of a weakly monotonic Minkowski-endomorphism $\Phi$, we have $\Phi \in \operatorname{End}_{\mathrm{M}}^{*}\left(\mathcal{K}^{d}\right)$ if and only if

$$
\tilde{\mu}([-1,1])=\lambda_{0}[\Phi]>0
$$

and

$$
\int_{-1}^{1} \alpha d \tilde{\mu}(\alpha)=\lambda_{1}[\Phi]=1
$$

Proof of Theorem 1.8. Consider $\Phi \in \operatorname{End}_{\mathrm{M}}^{*}\left(\mathcal{K}^{d}\right)$ for some $d \geq 3$. According to Theorem 1.4. the mixing measure $\tilde{\mu}$ of $\Phi$ is positive up to addition of a linear measure $\tilde{\nu}$ (and the sum $\tilde{\mu}+\tilde{\nu}$ is non-zero, as $(\tilde{\mu}+\tilde{\nu})([-1,1])=\tilde{\mu}([-1,1])>$ $0)$. Theorem 1.7 implies that there is a Blaschke-endomorphism $\Phi^{*}$ with mixing measure $\tilde{\mu}$. That (1.9) holds for this choice of $\Phi^{*}$ follows from (1.8), Fubini's theorem and (2.20). $\Phi^{*}$ is the only Blaschke-endomorphism satisfying (1.9), as any convex body $M \in \mathcal{K}_{0}^{d}$ is uniquely determined by the function

$$
K \mapsto V(K, M[d-1]), \quad K \in \mathcal{K}^{d} .
$$


We have seen that $\Lambda: \Phi \mapsto \Phi^{*}$ is well defined and that the mixing measure of $\Phi$ is a mixing measure of $\Phi^{*}$. To show Theorem [1.8 ii), it remains to show that $\Lambda$ is bijective.

To show surjectivity, let $\Psi$ be a Blaschke-endomorphism and $\tilde{\mu} \neq 0$ a mixing measure of $\Psi$. Without loss of generality, we may assume

$$
\int_{-1}^{1} \alpha d \tilde{\mu}(\alpha)=1
$$

as we may add an arbitrary linear measure to $\tilde{\mu}$. The measure $\tilde{\mu}$ is the mixing measure of a weakly monotonic Minkowski-endomorphism $\Phi$, due to Theorem 1.4. $\Phi \in \operatorname{End}_{\mathrm{M}}^{*}\left(\mathcal{K}^{d}\right)$, as (3.19) and (3.20) hold. (1.9) holds with this choice of $\Phi$, as the mixing measures of $\Phi$ and $\Psi$ coincide.

To show injectivity of $\Lambda$, let $\Phi, \chi \in \operatorname{End}_{\mathrm{M}}^{*}\left(\mathcal{K}^{d}\right)$ with $\Phi^{*}=\chi^{*}$ be given. Fix $K \in \mathcal{K}_{0}^{d}$. Then $\Phi K, \chi K \in \mathcal{K}_{0}^{d}$, as $\Phi$ and $\chi$ are non-degenerate. (1.9) implies

$$
V(\Phi K, M[d-1])=V(\chi K, M[d-1])
$$

for all $M \in \mathcal{K}_{0}^{d}$, so $\Phi$ and $\chi$ coincide on $\mathcal{K}_{0}^{d}$. Continuity and $T_{d}$-equivariance of both maps now imply $\Phi=\chi$ on $\mathcal{K}^{d}$. We have shown Theorem 1.8 ii).

Corollaries 3.1 and 3.6 imply Theorem [1.8 v), as $\lambda_{1}[\Phi]=1 \neq 0$ for all $T_{d^{-}}$ equivariant Minkowski-endomorphisms $\Phi$. The proofs of i) and ii) are straightforward.

Proof of Theorem 1.10. Without loss of generality we may assume that $\Phi$ is monotonic, i.e. its mixing measure $\tilde{\mu}$ is positive. Assume that $K \in \mathcal{K}^{d}$ is not a singleton and satisfies

$$
\Phi K=\beta K+x
$$

for some $\beta \in \mathbb{R}$ and $x \in \mathbb{R}^{d}$. Let $h(K, \cdot) \sim \sum_{n=0}^{\infty} h_{n}(K, \cdot)$ be the Fourier expansion of the support function of $K$. (3.21) and the multiplier property of $\Phi$ implies

$$
\beta h(K, \cdot)+\langle x, \cdot\rangle=h(\Phi K, \cdot)=\sum_{n=0}^{\infty} \lambda_{n}[\Phi] h_{n}(K, \cdot) .
$$

This gives $\beta=\lambda_{n}[\Phi]$ for all $n \neq 1$ with $h_{n}(K, \cdot) \neq 0$. As $K$ is not a singleton, $h_{0}(K, \cdot)=\frac{1}{2} \bar{w}(K) \neq 0$, so $\beta=\lambda_{0}[\Phi]$ is the radius of the ball $\Phi B^{d}$.

$\Phi$ was assumed to be non-trivial, so $\tilde{\mu}$ has mass in the open interval $(-1,1)$. (2.5) implies

$$
\left|\lambda_{n}[\Phi]\right| \leq \int_{-1}^{1}\left|P_{n}^{d}(\alpha)\right| d \tilde{\mu}(\alpha)<\lambda_{0}[\Phi]=\beta
$$

for all $n=2,3,4, \ldots$ This gives $h(K, \cdot)=h_{0}(K, \cdot)+h_{1}(K, \cdot)$, and $K$ is a ball.

The proof of Theorem 1.11 is almost literally the same, so we may omit it here.

\section{REFERENCES}

[1] Berg, C. (1969) Corps convexes et potentiels sphériques, Danske Vid. Selsk. Mat.-Fys. Medd. 37, 6. MR0254789 (40:7996)

[2] DunkL, C.F. (1966) Operators and harmonic analysis on the sphere, Trans. Amer. Math. Soc. 125, 250-263. MR0203371 (34:3224)

[3] Erdélyi, A., Magnus, W., Oberhettinger F., Tricomi, F.G. (1953) Higher Transcendental Functions, Vol. 2, McGraw-Hill, New York.

[4] Fallert, H., Goodey, P., Weil, W. (1997) Spherical projections and centrally symmetric sets, Adv. Math. 129, No. 2, 301-322. MR1462736(98j:52005) 
[5] Groemer, H. (1996) Geometric Applications of Fourier Series and Spherical Harmonics, Encyclopedia of Mathematics and its Applications, Vol. 61, Cambridge University Press, Cambridge. MR1412143 (97j:52001)

[6] Goodey, P. (1998) Minkowski sums of projections of convex bodies, Mathematika 45, 253268. MR,1695718 (2000e:52002)

[7] Goodey, P., WeIL, W. (1993) Zonoids and generalizations, in: Handbook of convex geometry, eds. P.M. Gruber and J.M. Wills, Elsvier Sci. Publ. B.V., 1297-1326. MR.1243010 (95g:52015)

[8] Goodey, P., Jiang, W. (2000) Minkowski sums of three dimensional projections of convex bodies, Suppl. Rend. Circ. Mat. Palermo, II. Ser. 65, 105-119. MR.1809148 (2001j:52005)

[9] Goodey, P., Kiderlen, M., Weil, W. (1998) Section and projection means of convex bodies, Mh. Math. 126, 37-54. MR1633259 (99d:52004)

[10] Hug, D, Last, G. (2000) On support measures in Minkowski spaces and contact distributions in stochastic geometry, Ann. of Probab. 28, No. 2, 796-850. MR.1782274 (2001g:60023)

[11] Kiderlen, M. (1999) Schnittmittelungen und äquivariante Endomorphismen konvexer Körper, Ph.D. Thesis, University of Karlsruhe.

[12] Kiderlen, M. (2004) Determination of a convex body from Minkowski sums of its projections, J. London Math. Soc. 70, No. 2, 529-544. MR2078909

[13] Seeley, R.T. (1966) Spherical harmonics, Amer. Math. Monthly 73, 115-121. MR0201695 $(34: 1577)$

[14] SCHNEIDER, R. (1969) Functions on a sphere with vanishing integrals over certain subspheres, J. Math. Anal. Appl. 26, 381-384. MR0237723 (38:6004)

[15] SChNeider, R. (1974) Equivariant endomorphisms of the space of convex bodies, Trans. Am. Math. Soc. 194, 53-78. MR0353147 (50:5633)

[16] Schneider, R. (1974) Bewegungsäquivariante, additive und stetige Transformationen konvexer Bereiche, Arch. Math. 25, 303-312. MR0344999 (49:9738)

[17] Schneider, R. (1977) Rekonstruktion eines konvexen Körpers aus seinen Projektionen, Math. Nachr. 79, 325-329. MR0500538(58:18147)

[18] Schneider, R. (1993) Convex Bodies: The Brunn-Minkowski Theory, Encyclopedia of Mathematics and its Applications, Vol. 44, Cambridge University Press, Cambridge. MR 1216521 (94d:52007)

[19] Schneider, R., Weil, W. (1992) Integralgeometrie, Teubner, Stuttgart. MR.1203777 (94c:52003)

[20] Schwartz, L. (1957) Théorie des distributions, Hermann, Paris. MR0209834 (35:730)

[21] Spriestersbach, K.K. (1998) Determination of a convex body from the average of projections and stability results, Math. Proc. Camb. Philos. Soc. 123, 561-569. MR1608001(99b:52010)

[22] WeIL, W. (1976) Centrally symmetric convex bodies and distributions, Israel J. Math. 24, 352-367. MR0420436 (54:8450)

[23] Weil, W. (1997) On the mean shape of particle processes, Adv. Appl. Probab. 29, 890-908. MR:1484773 (99b:60015)

Mathematisches Institut II, Universität Karlsruhe, D-76128 Karlsruhe, Germany E-mail address: kiderlen@imf.au.dk

Current address: Department of Mathematical Sciences, University of Aarhus, Ny Munkegade, Bgn. 1530, DK-8000 Aarhus C, Denmark 